\title{
A novel comprehensive analysis of the refinements of Hermite-Hadamard type integral inequalities involving spe- cial functions
}

\author{
Muhammad Tariqa,*, Hijaz Ahmad ${ }^{\mathrm{b}, \mathrm{c}}$, Soubhagya Kumar Sahood, Lama Sh. Aljoufie,f, Sher Khan Awan ${ }^{\mathrm{a}}$ \\ a Department of Basic Sciences and Related Studies, Mehran University of Engineering and Technology, Jamshoro 76062, Pakistan. \\ ${ }^{b}$ Department of Computer Engineering, Biruni University, Istanbul 34025, Turkey. \\ ' Section of Mathematics, International Telematic University Uninettuno, Corso Vittorio Emanuele II, 39, 00186, Roma, Italy. \\ ${ }^{d}$ Department of Mathematics, Institute of Technical Education and Research, Siksha 'O' Anusandhan University, Bhubaneswar 751030, \\ India. \\ ${ }^{e}$ Deanship of Common First Year, Jouf University, P.O.Box 2014 Sakaka, Saudi Arabia. \\ ${ }^{f}$ Basic Sciences Research Unit, Jouf University, P.O.Box 2014 Sakaka, Saudi Arabia.
}

\begin{abstract}
The main objective of this article is to employ the concept of preinvexity to establish some new inequalities. In addition, we discuss some algebraic properties and examples of the generalized preinvex function. With the help of this new relation, we present new version of Hermite-Hadamard inequality and its some of its refinements using fundamental inequalities like Hölder, power-mean, Hölder-İscan, and improved power-mean inequality. These results are speculations of various recently known outcomes. The immeasurable concepts and tools of this paper may invigorate and revitalize for additional research in this mesmerizing and absorbing field.
\end{abstract}

Keywords: s-type convex function, preinvex function, m-preinvex function, Hölder's inequality, Hölder-İscan integral inequality, power mean inequality, improved power-mean integral inequality.

2020 MSC: 26A51, 26A33, 26D07, 26D10, $26 \mathrm{D} 15$.

(C)2022 All rights reserved.

\section{Introduction}

The theory of convexity presents a very impressive, remarkable, and mesmerizing field for research activities. This theory provides us amazing framework to initiate and develop numerical tools to tackle and study complicated problems in mathematics. This theory has a large number of potential applications in many different areas of sciences, such as information theory, coding theory, physics, optimization, engineering, and inequality theory. Due to this reason, this field has been very inspirational and popular

\footnotetext{
*Corresponding author

Email addresses: captaintariq2187@gmail.com (Muhammad Tariq), hijaz555@gmail.com (Hijaz Ahmad), soubhagyalulu@gmail.com (Soubhagya Kumar Sahoo), lamashuja11@gmail.com (Lama Sh. Aljoufi), sher.khan@faculty.muet.edu.pk (Sher Khan Awan)
}

doi: $10.22436 /$ jmcs.026.04.02

Received: 2021-09-03 Revised: 2021-09-23 Accepted: 2021-11-23 
among researchers. To gain detailed knowledge about convexity, we suggest readers to see the references $[1,12,18,22,24,25,30]$.

The concept of convex analysis has played crucial and consequential role in the extensions and generalizations of the theory of inequality. The theory of inequality along with convexity property plays an essential part in the present-day mathematical investigations. In addition, the modern and amazing view point on the theory of inequality always offers to proliferate ideas and fruitful applications in every field and branch of pure an applied sciences, for example, coding theory, geometric function theory, impulsive diffusion equations, numerical analysis, and fractional calculus. For the attention of the readers, we suggest to see the references [5-8].

Toader [27], for the first time introduced m-convex functions. After Toader, Latif [15] and Kalsoom [9] polished the idea of $m$-convexity and explored in the form of further generalizations namely $m$ preinvexity. Recently İşcan (see [23]) introduced s-type convex function. The s-type convex functions are very useful for the generation of Julia and Mandelbrot sets involving cubic and quadratic polynomial (see $[13,14])$. The awe-inspiring techniques and majestic tools of this article may excite and energize for further research activities in this direction.

Motivated by the ongoing research activities, the principal objective of this article is to present a new class of preinvex function called $s$-type m-preinvex function and $s$-type $(\alpha, m)$-preinvex functions before the readers. We explore some algebraic properties and examples in the manner of newly introduced definitions. Some new versions of Hermite-Hadamard $(\mathrm{H}-\mathrm{H})$ inequality and its refinements are investigated in the support of newly discussed concepts.

\section{Preliminaries}

In this section, we remember some known concepts. Mititelu [16] introduced the notion of invex set, which is defined as follows

Definition 2.1. Let $\Psi: \mathcal{J} \times \mathcal{J} \neq \emptyset \rightarrow \mathcal{R}$ be a real valued function and for every $\zeta, \xi \in \mathcal{J}$ and $\omega \in[0,1]$, then $\mathcal{J}$ is known as invex with respect to $\eta(.,$.$) if \xi+\omega \eta(\zeta, \xi) \in \mathcal{J}$.

We clearly see that, if we choose $\eta(\zeta, \xi)=\zeta-\xi$, then we attain classical convexity. Therefore, every convex set is an invex with respect to $\eta(\zeta, \xi)=\zeta-\xi$, but its converse is not true in general, means that $\exists$ invex sets which are not convex (see $[2,16]$ ).

Mathematicians namely Weir and Mond [29] used the idea of invex set and to perform and explore the idea of preinvexity.

Definition 2.2. Suppose an invex set $\mathcal{J} \neq \emptyset \in \mathcal{R}$ with respect to $\Psi: \mathcal{J} \times \mathcal{J} \neq \emptyset \rightarrow \mathcal{R}$. Then the mapping $\Psi: \mathcal{J} \rightarrow \mathcal{R}$ is known as preinvex with respect to $\eta$, if

$$
\Psi(\xi+\omega \eta(\zeta, \xi)) \leqslant(\omega) \Psi(\zeta)+(1-\omega) \Psi(\xi), \quad \forall \zeta, \xi \in \mathbb{X}, \omega \in[0,1] .
$$

In 2007, Noor [21] for the first time introduced the idea of preinvex function to investigate $\mathrm{H}-\mathrm{H}$ type inequality, which is given as follows.

Theorem 2.3. Suppose $\Psi: \mathcal{J}=[\zeta, \zeta+\eta(\xi, \zeta)] \rightarrow(0, \infty)$ be a preinvex functions on $\mathbb{A}^{\circ}$ and $\zeta, \xi \in \mathcal{J}$ with $\zeta<\zeta+\eta(\zeta, \xi)$. Then

$$
\Psi\left(\frac{2 \zeta+\eta(\xi, \zeta)}{2}\right) \leqslant \frac{1}{\eta(\xi, \zeta)} \int_{\zeta}^{\zeta+\eta(\xi, \zeta)} \Psi(x) d x \leqslant \frac{\Psi(\zeta)+\Psi(\xi)}{2} .
$$

In the year 2011, another team of mathematicians namely Barani, Gahazanfari, and Dragomir worked on the concept of preinvex function in the published article [4] and for the first time explored and established $\mathrm{H}-\mathrm{H}$ type inequality, which is given as follows. 
Theorem 2.4. Suppose an open invex subset $\mathcal{J} \subseteq \mathcal{R}$ with respect to $\eta: \mathcal{J} \times \mathcal{J} \rightarrow \mathcal{R}$. Let mapping $\Psi: \mathcal{J} \rightarrow \mathcal{R}$ be differentiable. If $\left|\Psi^{\prime}\right|$ is preivex on $\mathcal{J}$ and $\forall \zeta, \xi \in \mathcal{J}$ with $\eta(\xi, \zeta) \neq 0$, then

$$
\left|\frac{\Psi(\zeta)+\Psi(\zeta+\eta(\xi, \zeta)}{2}-\frac{1}{\eta(\xi, \zeta)} \int_{\zeta}^{\zeta+\eta(\xi, \zeta)} \Psi(x) d x\right| \leqslant \frac{|\eta(\xi, \zeta)|}{8}[\Psi(\zeta)+\Psi(\xi)] .
$$

Later on, different authors worked and collaborated on the concept of preinvex functions as presented in the given articles $[3,10,19,20]$. Mohan and Neogy [17], first time used the condition-C, which is given as follows.

Condition-C: Suppose that for any $\zeta, \xi \in \mathcal{J}, \omega \in[0,1]$ and an open invex subset $\mathcal{J} \subset \mathrm{R}^{\mathrm{n}}$ with respect to $\eta: \mathcal{J} \times \mathcal{J} \rightarrow \mathcal{R}$, then

$$
\eta(\xi, \xi+\omega \eta(\zeta, \xi))=-\omega \eta(\zeta, \xi), \quad \eta(\zeta, \xi+\omega \eta(\zeta, \xi))=(1-\omega) \eta(\zeta, \xi) .
$$

For any $\zeta, \xi \in \mathcal{J}$ and $\omega_{1}, \omega_{2} \in[0,1]$ from condition $C$, we have

$$
\eta\left(\xi+\omega_{2} \eta(\zeta, \xi), \xi+\omega_{1} \eta(\zeta, \xi)\right)=\left(\omega_{2}-\omega_{1}\right) \eta(\zeta, \xi) .
$$

From the last current decade, many mathematicians polished and worked on the concept of preinvex functions in a different ways. Recently Latif [15] investigated first time the idea of generalized m-preinvex and $(\alpha, m)$-preinvex function, which are given as follows.

Definition 2.5. Suppose a function $\Psi: \mathcal{J} \rightarrow \mathcal{R}$ on $\mathcal{J} \subseteq\left[0, \mathrm{~b}^{*}\right], \mathrm{b}^{*}>0$ and $\mathrm{m} \in(0,1]$, then $\Psi$ is known as m-preinvex with respect to $\eta$, if

$$
\Psi(\xi+\omega \eta(\zeta, \xi)) \leqslant(1-\omega) \Psi(\xi)+m \omega \Psi\left(\frac{\zeta}{m}\right),
$$

holds for every $\zeta, \xi \in \mathcal{J}, \omega \in[0,1]$.

Definition 2.6. Suppose $\Psi: \mathcal{J} \rightarrow \mathcal{R}$ on $\mathbb{X} \subseteq\left[0, y^{*}\right], y^{*}>0$, then $\Psi$ is known as $(\alpha, m)$-preinvex with respect to $\eta$ fixed $(\alpha, m) \in(0,1] \times(0,1]$, if

$$
\Psi(\xi+\omega \eta(\zeta, \xi)) \leqslant\left(1-\omega^{\alpha}\right) \Psi(\xi)+m \omega^{\alpha} \Psi\left(\frac{\zeta}{m}\right),
$$

holds for every $\zeta, \xi \in \mathcal{J}, \omega \in[0,1]$.

İşcan [23], introduced s-type convex function, which is given as next.

Definition 2.7. A function $\Psi: \mathcal{J} \rightarrow \mathcal{R}$ is said to be a s-type convex function if for every $\zeta, \xi \in \mathcal{J}, s \in[0,1]$ and $\omega \in[0,1]$,

$$
\Psi(\omega \zeta+(1-\omega) \xi) \leqslant[1-(s(1-\omega))] \Psi(\zeta)+[1-s \omega] \Psi(\xi) .
$$

Skala [26], for the first time introduced and explored similarly ordered, which is given as follows.

Definition 2.8. An inequality of the form

$$
(\Psi(\zeta)-\Psi(\xi))(\Phi(\zeta)-\Phi(\xi)) \geqslant 0, \quad \forall \zeta, \xi \in \mathcal{R}
$$

is called similarly ordered.

Attributable to the previously mentioned pattern and propelled by the continuous activities, the paper is structured as follows. After discussing some preliminaries about preinvexity in Sections 1 and 2, we introduce and investigate the presented concept about $s$-type m-preinvex and s-type $(\alpha, m)$-preinvex functions in Section 3. In Section 4, we derive the novel version of $\mathrm{H}-\mathrm{H}$ type inequality. In section 5, based on a established integral identity, we present estimations of $\mathrm{H}-\mathrm{H}$ type inequality via newly introduced ideas. 


\section{Generalized preinvexity and its algebraic properties}

The principal focus and aim of this section is to investigate and explore new notions involving new class of convex functions namely s-type m-preinvex and s-type $(\alpha, m)$-preinvex function and also to discuss some algebraic properties in the manner of these newly introduced definitions.

Definition 3.1. Suppose a function $\Psi: \mathcal{J} \rightarrow \mathcal{R}$ on $\mathcal{J} \subseteq\left[0, b^{*}\right], b^{*}>0$ and $m \in(0,1]$. Then the function $\Psi$ is known as s-type m-preinvex with respect to $\eta$, if

$$
\Psi(\xi+\omega \eta(\zeta, \xi)) \leqslant(1-s \omega) \Psi(\xi)+(1-(s(1-\omega))) m \Psi\left(\frac{\zeta}{m}\right),
$$

holds for every $\zeta, \xi \in \mathbb{X}, \omega \in[0,1]$, and $s \in[0,1]$.

\section{Remark 3.2.}

(i) Choosing $s=m=1$ in Definition 3.1, it reduces to preinvex function which was introduced by Weir and Mond [29].

(ii) Choosing $m=1$ and $\eta(\zeta, \xi)=\zeta-\xi$ in Definition 3.1, it reduces to s-type convex function which was presented by İşcan et al. [23].

(iii) Choosing $s=m=1$ and $\eta(\zeta, \xi)=\zeta-\xi$ in Definition 3.1, it reduces to convex function which was introduced and discussed by Niculescu et al. [18].

Definition 3.3. Suppose a function $\Psi: \mathbb{X} \rightarrow \mathcal{R}$ on $\mathbb{X} \subseteq\left[0, \mathrm{~b}^{*}\right], \mathrm{b}^{*}>0$ and $(\alpha, \mathrm{m}) \in(0,1] \times(0,1]$. Then the function $\Psi$ is known as s-type $(\alpha, m)$-preinvex with respect to $\eta$, if

$$
\Psi(\xi+\omega \eta(\zeta, \xi)) \leqslant\left(1-(s \omega)^{\alpha}\right) \Psi(\xi)+(1-(s(1-\omega)))^{\alpha} m \Psi\left(\frac{\zeta}{m}\right),
$$

holds for every $\zeta, \xi \in \mathbb{X}, \omega \in[0,1]$, and $s \in[0,1]$.

Remark 3.4.

(i) Choosing $s=\alpha=m=1$ in Definition 3.3, it reduces to preinvex function.

(ii) Choosing $\alpha=m=1$ and $\eta(\zeta, \xi)=\zeta-\xi$ in Definition 3.3, it reduces to s-type convex function.

(iii) Choosing $s=\alpha=m=1$ and $\eta(\zeta, \xi)=\zeta-\xi$ in Definition 3.3, it reduces to convex function.

Lemma 3.5. Suppose, $\forall s \in[0,1], m \in(0,1]$ and $\omega \in[0,1]$, then the following inequalities

$$
\omega \leqslant(1-(s(1-\omega))) \text { and } 1-\omega \leqslant 1-s \omega
$$

hold.

Proof. The proof is obvious.

Proposition 3.6. Suppose every $s \in[0,1], \mathrm{m} \in(0,1]$, and $\omega \in[0,1]$ holds, then every nonnegative m-preinvex function is an s-type m-preinvex function.

Proof. According to the Lemma 3.5 and definition of m-preinvexity, we have

$$
\Psi(\xi+\omega \eta(\zeta, \xi)) \leqslant(1-\omega) \Psi(\xi)+m \omega \Psi\left(\frac{\zeta}{m}\right) \leqslant(1-s \omega) \Psi(\xi)+(1-(s(1-\omega))) m \Psi\left(\frac{\zeta}{m}\right) .
$$

Proposition 3.7. Suppose $\omega \in[0,1], \mathrm{m} \in(0,1]$, and $\mathrm{s} \in[0,1]$, then every non-negative s-type $\mathrm{m}$-preinvex is an $(\mathrm{h}, \mathrm{m})$-preinvex function with the condition $\mathrm{h}(\omega)=(1-(\mathrm{s}(1-\omega)))$. 
Proof. From the property of s-type m-preinvex function and the mentioned condition $h(\omega)=(1-(s(1-\omega)))$, we have

$$
\Psi(\xi+\omega \eta(\zeta, \xi)) \leqslant(1-s \omega) \Psi(\xi)+(1-(s(1-\omega))) m \Psi\left(\frac{\zeta}{m}\right) \leqslant h(1-\omega) \Psi(\xi)+h m(\omega) \Psi\left(\frac{\zeta}{m}\right) .
$$

Now, we present examples for the new definitions.

Example 3.8. It is obvious that, every convex function is preinvex function. So using this concept, we say that, a non-negative convex function $\Psi(x)=e^{x}, \forall x \geqslant 0$ is $m$-preinvex function if $m=1$. According to Proposition 3.6, it is an s-type m-preinvex function.

Example 3.9. Let $\Psi(x): \mathbb{R}^{+} \rightarrow \mathbb{R}^{+}$is defined by

$$
\Psi(\tau)=\left\{\begin{array}{ll}
\tau+1, & 0 \leqslant \tau \leqslant 1, \\
1, & \tau>1,
\end{array} \quad \text { and } \quad \eta(\zeta, \xi)=\left\{\begin{array}{ll}
\zeta+\xi, & \zeta \leqslant \xi, \\
2(\zeta+\xi), & \zeta>\xi,
\end{array} \quad \forall \zeta, \xi \in \mathbb{R}^{+}=[0,+\infty) .\right.\right.
$$

The above non-negative function $\Psi(\tau)$ is a m-preinvex function, if $m=1$, but not a convex function. According to Proposition 3.6, it is s-type m-preinvex function on $\mathrm{X}$.

Now, we will explore and obtain some algebraic properties in the manner of the s-type m-preinvex functions.

Theorem 3.10. Suppose two mappings $\Psi, \Phi: \mathcal{J}=[\zeta, \xi] \rightarrow \mathcal{R}$ be s-type m-preinvex functions, then

(i) $\Psi+\Phi$;

(ii) $\mathrm{c} \Psi$,

are s-type m-preinvex functions.

Proof.

(i) $\forall \zeta, \xi \in \mathcal{J}, \omega \in[0,1], s \in[0,1]$ and $m \in(0,1]$, we attain

$$
\begin{aligned}
(\Psi+\Phi)(\xi+\omega \eta(\zeta, \xi))= & \Psi(\xi+\omega \eta(\zeta, \xi))+\Phi(\xi+\omega \eta(\zeta, \xi)) \\
\leqslant & (1-s \omega) \Psi(\xi)+(1-(s(1-\omega))) m \Psi\left(\frac{\zeta}{m}\right) \\
& +(1-s \omega) \Phi(\xi)+(1-(s(1-\omega))) m \Phi\left(\frac{\zeta}{m}\right) \\
= & (1-s \omega)[\Psi(\xi)+\Phi(\xi)]+(1-(s(1-\omega)))\left[m \Psi\left(\frac{\zeta}{m}\right)+m \Phi\left(\frac{\zeta}{m}\right)\right] \\
= & (1-s \omega)(\Psi+\Phi)(\xi)+(1-(s(1-\omega))) m(\Psi+\Phi)\left(\frac{\zeta}{m}\right) .
\end{aligned}
$$

(ii) $\forall \zeta, \xi \in \mathcal{J}, \omega \in[0,1], m \in(0,1], s \in[0,1]$ and $c \in \mathcal{R}(c \geqslant 0)$, we have

$$
\begin{aligned}
(\mathrm{c} \Psi)(\xi+\omega \eta(\zeta, \xi)) & \leqslant c\left[(1-s \omega) \Psi(\xi)+(1-(s(1-\omega))) \mathrm{m} \Psi\left(\frac{\zeta}{\mathrm{m}}\right)\right] \\
& =(1-\mathrm{s} \omega) \mathrm{c} \Psi(\xi)+(1-(\mathrm{s}(1-\omega))) \mathrm{cm} \Psi\left(\frac{\zeta}{\mathrm{m}}\right) \\
& =(1-\mathrm{s} \omega)(\mathrm{c} \Psi)(\xi)+(1-(\mathrm{s}(1-\omega))) \mathrm{m}(\mathrm{c} \Psi)\left(\frac{\zeta}{\mathrm{m}}\right) .
\end{aligned}
$$

Hence, the desired result is obtained. 
Theorem 3.11. Suppose for every $\omega \in[0,1], m \in(0,1], s \in[0,1]$ and $\Psi: \mathcal{J} \rightarrow \mathbb{Y}$ and $\Phi: \mathbb{Y} \rightarrow \mathcal{R}$ be an s-type $\mathrm{m}$-preinvex and increasing function, respectively. Then the composition of both functions, i.e., $\Phi \circ \Psi$ is an s-type m-preinvex function.

Proof. $\forall \zeta, \xi \in \mathcal{J}, m \in(0,1], \omega \in[0,1]$ and $s \in[0,1]$, we attain

$$
\begin{aligned}
(\Phi \circ \Psi)(\xi+\omega \eta(\zeta, \xi)) & =\Phi(\Psi(\xi+\omega \eta(\zeta, \xi))) \\
& \leqslant \Phi\left[(1-s \omega) \Psi(\xi)+(1-(s(1-\omega))) m \Psi\left(\frac{\zeta}{m}\right)\right] \\
& \leqslant(1-s \omega) \Phi(\Psi(\xi))+(1-(s(1-\omega))) m \Phi\left(m \Psi\left(\frac{\zeta}{m}\right)\right) \\
& =(1-s \omega)(\Phi \circ \Psi)(\xi)+(1-(s(1-\omega))) m^{2}(\Phi \circ \Psi)\left(\frac{\zeta}{m}\right) .
\end{aligned}
$$

Hence, the desired result is obtained.

Remark 3.12.

(i) If we set $s=1$ in the above Theorem 3.11, we attain the inequality

$$
(\Phi \circ \Psi)(m \xi+\omega \eta(\zeta, \xi, m)) \leqslant(1-\omega)(\Phi \circ \Psi)(\xi)+\omega m^{2}(\Phi \circ \Psi)\left(\frac{\zeta}{m}\right) .
$$

(ii) If we set $\mathrm{m}=\mathrm{s}=1$ in the above Theorem 3.11, then as a result, we attain the inequality

$$
(\Phi \circ \Psi)(\xi+\omega \eta(\zeta, \xi)) \leqslant(1-\omega)(\Phi \circ \Psi)(\xi)+\omega(\Phi \circ \Psi)(\zeta) .
$$

(iii) Putting $\mathrm{m}=1$ and $\eta(\zeta, \xi, \mathrm{m})=\zeta-\mathrm{m} \xi$ in the above Theorem 3.11, we have

$$
(\Phi \circ \Psi)(\omega \zeta+(1-\omega) \xi) \leqslant(1-s \omega)(\Phi \circ \Psi)(\xi)+(1-(s(1-\omega))(\Phi \circ \Psi)(\zeta) .
$$

Theorem 3.13. Suppose for every $\omega \in[0,1], m \in(0,1], s \in[0,1], 0<\zeta<\xi, \Psi_{j}: \mathcal{J}=[\zeta, \xi] \rightarrow \mathcal{R}^{+}$be a family of s-type $\mathrm{m}$-preinvex function and $\Psi(\mathrm{u})=\sup _{\mathfrak{j}} \Psi_{\mathfrak{j}}(\mathfrak{u})$, then $\Psi$ is an s-type $\mathrm{m}$-preinvexity and $\mathrm{U}=\{\tau \in[\zeta, \xi]$ : $\left.\Psi\left(\tau_{i}\right)<\infty\right\}$ is an interval.

Proof.

$$
\begin{aligned}
\Psi(\xi+\omega \eta(\zeta, \xi))=\sup _{j} \Psi_{j}(\xi+\omega \eta(\zeta, \xi)) & \leqslant(1-s \omega) \sup _{j} \Psi_{j}(\xi)+(1-(s(1-\omega))) \operatorname{msup}_{j} \Psi_{j}\left(\frac{\zeta}{m}\right) \\
& =(1-s \omega) \Psi(\xi)+(1-(s(1-\omega))) m \Psi\left(\frac{\zeta}{m}\right)<\infty .
\end{aligned}
$$

This is the required proof.

Theorem 3.14. Suppose for every $\omega \in[0,1], m \in(0,1], s \in[0,1]$, and $\Psi_{i}: \mathcal{R}^{n} \rightarrow \mathcal{R}$ is an s-type m-preinvex function, then the set $\mathbb{M}=\left\{\tau \in \mathcal{R}: \Psi_{i}(\tau) \leqslant 0, i=1,2,3, \ldots, n\right\}$ is an m-invex set.

Proof. Since

$$
\Psi_{i}(\xi+\omega \eta(\zeta, \xi)) \leqslant(1-s \omega) \Psi(\xi)+(1-(s(1-\omega))) m \Psi\left(\frac{\zeta}{m}\right),
$$

when $\zeta, \xi \in \mathbb{M}$, we know $\Psi_{\mathfrak{i}}(\zeta) \leqslant 0$ and $\Psi_{\mathfrak{i}}(\xi) \leqslant 0$, from the above inequality, it yields that

$$
\Psi_{i}(\xi+\omega \eta(\zeta, \xi)) \leqslant 0, \quad i=1,2,3, \ldots, n .
$$

That is, $\xi+\omega \eta(\zeta, \xi) \in \mathbb{M}$. Hence, $\mathbb{M}$ is an m-invex set. 
Theorem 3.15. Suppose for every $\omega \in[0,1], m \in(0,1], s \in[0,1]$, and $\Psi: \mathcal{J} \subseteq \mathcal{R}^{n} \rightarrow \mathcal{R}$ is s-type $m$-preinvex function on $\mathrm{m}$-invex set $\mathcal{J}$, then $\Psi$ is also generalized quasi $\mathrm{m}$-preinvex function on $\mathrm{J}$.

Proof. We assume that $m \Psi\left(\frac{\zeta}{m}\right) \leqslant \Psi(\xi)$, then for all $\zeta, \xi \in \mathcal{J}$, we have

$$
\begin{aligned}
\Psi(\xi+\omega \eta(\zeta, \xi)) & \leqslant(1-s \omega) \Psi(\xi)+(1-(s(1-\omega))) m \Psi\left(\frac{\zeta}{m}\right) \\
& \leqslant[(1-(s(1-\omega)))+(1-s \omega)] \Psi(\xi) \leqslant \Psi(\xi) .
\end{aligned}
$$

In the same manner, let $\Psi(\xi) \leqslant m \Psi\left(\frac{\zeta}{m}\right)$, for all $\zeta, \xi \in \mathcal{J}$, we can also get

$$
\Psi(\xi+\omega \eta(\zeta, \xi)) \leqslant m \Psi\left(\frac{\zeta}{m}\right) .
$$

Consequently,

$$
\Psi(\xi+\omega \eta(\zeta, \xi)) \leqslant \max \{\Psi(\zeta), \Psi(\xi)\}
$$

This is the required proof.

Theorem 3.16. Suppose for every $\omega \in[0,1], s \in[0,1]$ and $m \in(0,1], \Psi: \mathcal{R}_{\circ} \rightarrow \mathbb{R}_{\circ}$ be an s-type m-preinvex function with respect to $\eta: \mathbb{R}_{\circ} \times \mathbb{R}_{\circ} \times(0,1] \rightarrow \mathbb{R}_{\circ}$. Suppose that, $\eta$ is monotone increasing and $\Psi$ is monotone decreasing regarding $m$ for fixed $\zeta, \xi \in \mathbb{R}_{\circ}$ and $m_{1} \leqslant m_{2}\left(m_{1}, m_{2} \in(0,1]\right)$. Suppose $\Psi$ is a s-type $m_{1}$-preinvex function, then $\Psi$ is a s-type $\mathrm{m}_{2}$-preinvex function.

Proof. Employing the definition of s-type $m_{1}$-preinvexity

$$
\Psi(\xi+\omega \eta(\zeta, \xi)) \leqslant(1-s \omega) \Psi(\xi)+(1-(s(1-\omega))) m_{1} \Psi\left(\frac{\zeta}{m_{1}}\right) .
$$

Combining $\Psi$ with $\eta$ regarding $m$ for some fixed $\zeta, \xi \in \mathbb{R}_{\circ}$ and $m_{1} \leqslant m_{2}$, it follows

$$
(1-s \omega) \Psi(\xi)+(1-(s(1-\omega))) m_{1} \Psi\left(\frac{\zeta}{m_{1}}\right) \leqslant(1-s \omega) \Psi(\xi)+(1-(s(1-\omega))) m_{2} \Psi\left(\frac{\zeta}{m_{2}}\right) .
$$

Finally, we have

$$
\Psi(\xi+\omega \eta(\zeta, \xi)) \leqslant(1-s \omega) \Psi(\xi)+(1-(s(1-\omega))) m_{2} \Psi\left(\frac{\zeta}{m_{2}}\right)
$$

Hence, the above inequality shows the definition of $s$-type $m_{2}$-preinvexity.

Theorem 3.17. Suppose $\omega \in[0,1], \mathrm{m} \in(0,1], \mathrm{s} \in[0,1]$ and $\Psi, \Phi: \mathcal{J}=[\zeta, \xi] \rightarrow \mathcal{R}$. If $\Psi, \Phi$ be two similarly ordered, s-type m-preinvex functions with the condition $[1-(\mathrm{s}(1-\omega))]+[1-\mathrm{s} \omega] \leqslant 1$, then $\Psi \Phi$ is s-type mpreinvex function.

Proof.

$$
\begin{aligned}
& \Psi(\xi+\omega \eta(\zeta, \xi)) \Phi(\xi+\omega \eta(\zeta, \xi)) \\
& \leqslant {\left[(1-s \omega) \Psi(\xi)+(1-(s(1-\omega))) m \Psi\left(\frac{\zeta}{m}\right)\right]\left[(1-s \omega) \Phi(\xi)+(1-(s(1-\omega))) m \Phi\left(\frac{\zeta}{m}\right)\right] } \\
& \leqslant(1-s \omega)^{2} \Psi(\xi) \Phi(\xi)+(1-(s(1-\omega)))^{2} m^{2} \Psi\left(\frac{\zeta}{m}\right) \Phi\left(\frac{\zeta}{m}\right) \\
&+(1-(s(1-\omega)))(1-s \omega)\left[m \Psi\left(\frac{\zeta}{m}\right) \Phi(\xi)+\Psi(\xi) m \Phi\left(\frac{\zeta}{m}\right)\right] \\
& \leqslant(1-s \omega)^{2} \Psi(\xi) \Phi(\xi)+[1-(s(1-\omega))]^{2} m^{2} \Psi\left(\frac{\zeta}{m}\right) \Phi\left(\frac{\zeta}{m}\right)
\end{aligned}
$$




$$
\begin{aligned}
& +(1-(s(1-\omega)))(1-(s \omega))\left[\mathrm{m}^{2} \Psi\left(\frac{\zeta}{\mathrm{m}}\right) \Phi\left(\frac{\zeta}{\mathrm{m}}\right)+\Psi(\xi) \Phi(\xi)\right] \\
= & {\left[(1-s \omega) \Psi(\xi) \Phi(\xi)+(1-(s(1-\omega))) \mathrm{m}^{2} \Psi\left(\frac{\zeta}{\mathrm{m}}\right) \Phi\left(\frac{\zeta}{\mathrm{m}}\right)\right]((1-(s(1-\omega)))+(1-s \omega)) } \\
\leqslant & (1-s \omega) \Psi(\xi) \Phi(\xi)+(1-(s(1-\omega))) \mathrm{m}^{2} \Psi\left(\frac{\zeta}{\mathrm{m}}\right) \Phi\left(\frac{\zeta}{\mathrm{m}}\right) .
\end{aligned}
$$

This is the required proof.

Remark 3.18. If $m=1$ and $\eta(\zeta, \xi, m)=\zeta-m \xi$ in Theorem 3.17, then as a result, we attain the inequality

$$
\Psi(\omega \zeta+(1-\omega) \xi) \Phi(\omega \zeta+(1-\omega) \xi) \leqslant[1-(s(1-\omega))] \Psi(\zeta) \Phi(\zeta)+[1-s \omega] \Psi(\xi) \Phi(\xi) .
$$

Theorem 3.19. Suppose mappings $\Psi, \Phi: \mathcal{J}=[\zeta, \xi] \rightarrow \mathcal{R}$ be two s-type $(\alpha, \mathrm{m})$-preinvex functions, then

(i) $\Psi+\Phi$ is s-type $(\alpha, m)$-preinvex function;

(ii) $\mathrm{c} \Psi$ is an s-type $(\alpha, \mathrm{m})$-preinvex function.

Proof.

(i) $\forall \zeta, \xi \in \mathcal{J}, \omega \in[0,1], s \in[0,1]$, and $(\alpha, m) \in(0,1] \times(0,1]$, we attain

$$
\begin{aligned}
(\Psi+\Phi)(\xi+\omega \eta(\zeta, \xi))= & \Psi(\xi+\omega \eta(\zeta, \xi))+\Phi(\xi+\omega \eta(\zeta, \xi)) \\
\leqslant & \left(1-(s \omega)^{\alpha}\right) \Psi(\xi)+(1-(s(1-\omega)))^{\alpha} m \Psi\left(\frac{\zeta}{m}\right) \\
& +\left(1-(s \omega)^{\alpha}\right) \Phi(\xi)+(1-(s(1-\omega)))^{\alpha} m \Phi\left(\frac{\zeta}{m}\right) \\
= & \left(1-(s \omega)^{\alpha}\right)[\Psi(\xi)+\Phi(\xi)]+(1-(s(1-\omega)))^{\alpha}\left[m \Psi\left(\frac{\zeta}{m}\right)+m \Phi\left(\frac{\zeta}{m}\right)\right] \\
= & \left(1-(s \omega)^{\alpha}\right)(\Psi+\Phi)(\xi)+(1-(s(1-\omega)))^{\alpha} m(\Psi+\Phi)\left(\frac{\zeta}{m}\right) .
\end{aligned}
$$

(ii) $\forall \zeta, \xi \in \mathcal{J}, \omega \in[0,1], s \in[0,1],(\alpha, m) \in(0,1] \times(0,1]$, and $c \in \mathcal{R}(c \geqslant 0)$, we attain

$$
\begin{aligned}
(\mathrm{c} \Psi)(\xi+\omega \eta(\zeta, \xi)) & \leqslant c\left[\left(1-(s \omega)^{\alpha}\right) \Psi(\xi)+(1-(s(1-\omega)))^{\alpha} \mathrm{m} \Psi\left(\frac{\zeta}{\mathrm{m}}\right)\right] \\
& =\left(1-(s \omega)^{\alpha}\right) \mathrm{c} \Psi(\xi)+(1-(s(1-\omega)))^{\alpha} \mathrm{cm} \Psi\left(\frac{\zeta}{\mathrm{m}}\right) \\
& =\left(1-(\mathrm{s} \omega)^{\alpha}\right)(\mathrm{c} \Psi)(\xi)+(1-(s(1-\omega)))^{\alpha} \mathrm{m}(\mathrm{c} \Psi)\left(\frac{\zeta}{\mathrm{m}}\right) .
\end{aligned}
$$

This is the required proof.

Theorem 3.20. Suppose for every $\omega \in[0,1], s \in[0,1]$ and $(\alpha, m) \in(0,1] \times(0,1], \Psi: \mathcal{J} \rightarrow \mathbb{Y}$ and $\Phi: \mathbb{Y} \rightarrow \mathcal{R}$ be an s-type $(\alpha, \mathrm{m})$-preinvex and an increasing function, respectively. Then $\Phi \circ \Psi$ is a s-type $(\alpha, \mathrm{m})$-preinvex function.

Proof.

$$
\begin{aligned}
(\Phi \circ \Psi)(\xi+\omega \eta(\zeta, \xi)) & =\Phi(\Psi(\xi+\omega \eta(\zeta, \xi))) \\
& \leqslant \Phi\left[\left(1-(s \omega)^{\alpha}\right) \Psi(\xi)+(1-(s(1-\omega)))^{\alpha} m \Psi\left(\frac{\zeta}{m}\right)\right]
\end{aligned}
$$




$$
\begin{aligned}
& \leqslant\left(1-(s \omega)^{\alpha}\right) \Phi(\Psi(\xi))+(1-(s(1-\omega)))^{\alpha} m \Phi\left(m \Psi\left(\frac{\zeta}{m}\right)\right) \\
& =\left(1-(s \omega)^{\alpha}\right)(\Phi \circ \Psi)(\xi)+(1-(s(1-\omega)))^{\alpha} m^{2}(\Phi \circ \Psi)\left(\frac{\zeta}{m}\right) .
\end{aligned}
$$

Hence, the desired result is obtained.

Theorem 3.21. Suppose $\omega \in[0,1], \mathrm{s} \in[0,1],(\alpha, \mathrm{m}) \in(0,1] \times(0,1]$. Let $0<\zeta<\xi, \Psi_{\mathrm{j}}: \mathcal{J}=[\zeta, \xi] \rightarrow[0,+\infty)$ be a family of s-type $(\alpha, \mathrm{m})$-preinvex function and $\Psi(\mathrm{u})=\sup _{\mathfrak{j}} \Psi_{\mathfrak{j}}(\mathfrak{u})$. Then $\Psi$ is an s-type $(\alpha, \mathrm{m})$-preinvex function and $\mathrm{U}=\left\{\tau \in[\zeta, \xi]: \Psi\left(\tau_{i}\right)<\infty\right\}$ is an interval.

Proof.

$$
\begin{aligned}
\Psi(\xi+\omega \eta(\zeta, \xi))=\sup _{j} \Psi_{j}(\xi+\omega \eta(\zeta, \xi)) & \leqslant\left(1-(s \omega)^{\alpha}\right) \sup _{j} \Psi_{j}(\xi)+(1-(s(1-\omega)))^{\alpha} \operatorname{msup}_{j} \Psi_{j}\left(\frac{\zeta}{m}\right) \\
& =\left(1-(s \omega)^{\alpha}\right) \Psi(\xi)+(1-(s(1-\omega)))^{\alpha} m \Psi\left(\frac{\zeta}{m}\right)<\infty .
\end{aligned}
$$

Hence, the desired result is obtained.

\section{4. $\mathrm{H}-\mathrm{H}$ type inequality in the frame of s-type m-preinvex functions}

The main objective of this part is to investigate the new sort of $\mathrm{H}-\mathrm{H}$ type inequality in the mode of newly introduced idea namely s-type m-preinvex functions.

Theorem 4.1. Let $\mathbb{X}^{\circ} \subseteq \mathcal{R}$ be an open invex subset with respect to $\eta: \mathbb{X}^{\circ} \times \mathbb{X}^{\circ} \times(0,1] \rightarrow \mathcal{R}$ and let $\zeta, \xi \in \mathbb{X}^{\circ}, \zeta<$ $\xi$ with $\xi+\eta(\zeta, \xi) \leqslant \xi$. Suppose $\Psi:[\xi+\eta(\zeta, \xi), \xi]$ for all $m \in(0,1]$ and justifies Condition-C, then

$$
\Psi\left(\xi+\frac{1}{2} \eta(\zeta, \xi)\right) \leqslant \frac{\left(1-\frac{s}{2}\right)}{\eta(\zeta, \xi)}\left[\int_{\xi}^{\xi+\eta(\zeta, \xi)} m \Psi\left(\frac{x}{m}\right) d x+\int_{\frac{m \xi+\eta(\zeta, \xi)}{m}}^{\xi} \Psi(x) d x\right] \leqslant(2-s)\left[\Psi(\xi)+m \Psi\left(\frac{\zeta}{m}\right)\right] .
$$

Proof. Since $\zeta, \xi \in \mathbb{X}^{\circ}$ and for every $\omega \in[0,1], s \in[0,1]$ and $m \in(0,1]$, we attain $\xi+\omega \eta(\zeta, \xi) \in \mathbb{X}^{\circ}$. For the proof of the first inequality, putting $\omega=\frac{1}{2}$ in the Definition 3.1, and employing condition $C$ and integrating over $[0,1]$,

$$
\begin{aligned}
& \Psi(b+\omega \eta(a, b)) \leqslant(1-(s w)) \Psi(b)+(1-(s(1-w))) m \Psi\left(\frac{a}{m}\right), \\
& \Psi\left(b+\frac{1}{2} \eta(a, b)\right) \leqslant\left(1-\frac{s}{2}\right)\left[m \Psi\left(\frac{a}{m}\right)+\Psi(b)\right] .
\end{aligned}
$$

So, we put the value of $a=m \xi+\omega \eta(\zeta, \xi, m)$ and $m b=m \xi+(1-\omega) \eta(\zeta, \xi, m)$ in the above inequality, we get

$$
\Psi\left(b+\frac{1}{2} \eta(a, b)\right)=\Psi\left(\xi+(1-\omega) \eta(\zeta, \xi)+\frac{1}{2} \eta(\xi+\omega \eta(\zeta, \xi), \xi+(1-\omega) \eta(\zeta, \xi))\right) .
$$

Now by using Condition C, we have

$$
\begin{aligned}
& \eta(\xi+\omega \eta(\zeta, \xi), \xi+(1-\omega) \eta(\zeta, \xi))=(\omega-1+\omega) \eta(\zeta, \xi), \\
& \eta(\xi+\omega \eta(\zeta, \xi), \xi+(1-\omega) \eta(\zeta, \xi))=(2 \omega-1) \eta(\zeta, \xi) .
\end{aligned}
$$

Now, putting the value of $\eta$ in (4.1),

$$
\Psi\left(b+\frac{1}{2} \eta(a, b)\right)=\Psi\left(\xi+(1-\omega) \eta(\zeta, \xi)+\frac{1}{2}(2 \omega-1) \eta(\zeta, \xi)\right),
$$




$$
\begin{aligned}
& \Psi\left(b+\frac{1}{2} \eta(a, b)\right)=\Psi\left(\xi+\left(1-w+w-\frac{1}{2}\right) \eta(\zeta, \xi)\right), \\
& \Psi\left(y+\frac{1}{2} \eta(x, y)\right)=\Psi\left(\xi+\frac{1}{2} \eta(\zeta, \xi)\right) .
\end{aligned}
$$

Now

$$
\begin{aligned}
\Psi\left(\xi+\frac{1}{2} \eta(\zeta, \xi)\right) & \leqslant\left(1-\frac{s}{2}\right)\left[\int_{0}^{1} \mathrm{~m} \Psi\left(\frac{\xi+\omega \eta(\zeta, \xi)}{\mathrm{m}}\right) \mathrm{d} \omega+\int_{0}^{1} \Psi\left(\xi+\frac{(1-\omega)}{\mathrm{m}} \eta(\zeta, \xi)\right) \mathrm{d} \omega\right] \\
& \leqslant\left(1-\frac{s}{2}\right) \frac{1}{\eta(\zeta, \xi)}\left[\int_{\xi}^{\xi+\eta(\zeta, \xi)} \mathrm{m} \Psi\left(\frac{x}{\mathrm{~m}}\right) \mathrm{d} x+\int_{\frac{m \xi+\eta(\zeta, \xi)}{m}}^{\xi} \Psi(x) \mathrm{d} x\right] .
\end{aligned}
$$

For the proof of the second inequality, using the definition of s-type m-preinvexity, we have

$$
\begin{aligned}
\frac{1}{\eta(\zeta, \xi)}\left[\int_{\xi}^{\xi+\eta(\zeta, \xi)} \mathrm{m} \Psi\left(\frac{x}{\mathrm{~m}}\right) \mathrm{d} x+\int_{\frac{\mathrm{m} \xi+\eta(\zeta, \xi)}{\mathfrak{m}}}^{\xi} \Psi(x) \mathrm{d} x\right] \leqslant & {\left[\int_{0}^{1} \Psi(\xi+\omega \eta(\zeta, \xi)) d \omega+\int_{0}^{1} \Psi\left(\xi+\frac{(1-\omega)}{\mathrm{m}} \eta(\zeta, \xi)\right) \mathrm{d} \omega\right] } \\
\leqslant & \int_{0}^{1}(1-(s \omega)) \Psi(\xi) \mathrm{d} \omega+\int_{0}^{1}(1-(s(1-\omega))) \mathrm{m} \Psi\left(\frac{\zeta}{\mathrm{m}}\right) \mathrm{d} \omega \\
& +\int_{0}^{1}(1-(s(1-\omega))) \Psi(\xi) \mathrm{d} \omega+\int_{0}^{1}(1-s \omega) \mathrm{m} \Psi\left(\frac{\zeta}{\mathrm{m}}\right) \mathrm{d} \omega \\
& \leqslant\left(\frac{2-s}{2}\right)\left[\Psi(\xi)+\Psi(\xi)+\mathrm{m} \Psi\left(\frac{\zeta}{\mathrm{m}}\right)+\mathrm{m} \Psi\left(\frac{\zeta}{\mathrm{m}}\right)\right] \\
& \leqslant(2-s)\left[\Psi(\xi)+\mathrm{m} \Psi\left(\frac{\zeta}{\mathrm{m}}\right)\right] .
\end{aligned}
$$

This is the required proof.

Corollary 4.2. If we put $\mathrm{s}=\mathrm{m}=1$ and $\eta(\zeta, \xi)=\zeta-\xi$ in Theorem 4.1, then we get $\mathrm{H}-\mathrm{H}$ inequality in [11].

\section{Refinements of $\mathrm{H}-\mathrm{H}$ type inequality}

The main subject of this section is to present some estimations of $(\mathrm{H}-\mathrm{H})$ type inequality for s-type $m$-preinvex and s-type $(\alpha, m)$-preinvex functions.

Lemma 5.1 ([4]). Let $\Psi: \mathcal{J}^{\circ} \subseteq \mathcal{R} \rightarrow \mathcal{R}$ be a differentiable mapping on $\mathcal{J}^{\circ}, \zeta, \xi \in \mathcal{J}^{\circ}$ with $\zeta<\xi$. If $\Psi^{\prime} \in \mathrm{L}[\zeta, \xi]$, then

$$
-\frac{\Psi(\xi)+\Psi(\xi+\eta(\zeta, \xi))}{2}+\frac{1}{\eta(\zeta, \xi)} \int_{\xi}^{\xi+\eta(\zeta, \xi)} \Psi(x) d x=\frac{\eta(\zeta, \xi)}{2} \int_{0}^{1}(1-2 \omega) \Psi^{\prime}(\xi+\omega \eta(\zeta, \xi)) d \omega .
$$

Theorem 5.2. Suppose $\mathcal{J}^{\circ} \subseteq \mathcal{R}$ be an open invex subset with respect to $\eta: \mathcal{J}^{\circ} \times \mathcal{J}^{\circ} \rightarrow \mathcal{R}$ and $\zeta, \xi \in \mathcal{J}^{\circ}$ with $\xi+\eta(\zeta, \xi) \leqslant \xi$. Assume that mapping $\Psi: \mathcal{J}^{\circ} \rightarrow \mathcal{R}$ be differentiable such that $\Psi^{\prime} \in \mathrm{L}[\xi+\eta(\zeta, \xi), \xi]$. If $\left|\Psi^{\prime}\right|$ is s-type $m$-preinvex function on $[\xi+\eta(\zeta, \xi), \xi]$ and $\omega \in[0,1], s \in[0,1], m \in(0,1]$, then

$$
\left|\frac{\Psi(\xi)+\Psi(\xi+\eta(\zeta, \xi))}{2}-\frac{1}{\eta(\zeta, \xi)} \int_{\xi}^{\xi+\eta(\zeta, \xi)} \Psi(x) d x\right| \leqslant|\eta(\zeta, \xi)|\left(\frac{2-s}{4}\right) A\left(m\left|\Psi^{\prime}\left(\frac{\zeta}{m}\right)\right|,\left|\Psi^{\prime}(\xi)\right|\right),
$$

where $A$ is arithmetic mean.

Proof. Assume that for any $\omega \in[0,1]$ and $\zeta, \xi \in \mathcal{J}^{\circ}$, we have $\xi+\omega \eta(\zeta, \xi) \in \mathcal{J}^{\circ}$. Using Lemma 5.1, properties of modulus and definition of $\left|\Psi^{\prime}\right|$, we have 


$$
\begin{aligned}
& \left|\frac{\Psi(\xi)+\Psi(\xi+\eta(\zeta, \xi))}{2}-\frac{1}{\eta(\zeta, \xi)} \int_{\xi}^{\xi+\eta(\zeta, \xi)} \Psi(x) d x\right| \\
& \quad \leqslant\left|\frac{\eta(\zeta, \xi)}{2} \int_{0}^{1}(1-2 \omega) \Psi^{\prime}(\xi+\omega \eta(\zeta, \xi)) d \omega\right| \\
& \quad \leqslant \frac{|\eta(\zeta, \xi)|}{2} \int_{0}^{1}|1-2 \omega|\left((1-(s \omega))\left|\Psi^{\prime}(\xi)\right|+(1-(s(1-\omega))) \mathrm{m}\left|\Psi^{\prime}\left(\frac{\zeta}{\mathrm{m}}\right)\right|\right) \mathrm{d} \omega \\
& \quad \leqslant \frac{|\eta(\zeta, \xi)|}{2}\left(\left|\Psi^{\prime}(\xi)\right| \int_{0}^{1}|1-2 \omega|(1-(s \omega)) \mathrm{d} \omega+\mathrm{m}\left|\Psi^{\prime}\left(\frac{\zeta}{\mathrm{m}}\right)\right| \int_{0}^{1}|1-2 \omega|(1-(s(1-\omega))) \mathrm{d} \omega\right) \\
& \quad \leqslant \frac{|\eta(\zeta, \xi)|}{2}\left(\left|\Psi^{\prime}(\xi)\right|\left(\frac{2-s}{4}\right)+\left|\mathrm{m} \Psi^{\prime}\left(\frac{\zeta}{\mathrm{m}}\right)\right|\left(\frac{2-s}{4}\right)\right) \\
& \quad \leqslant|\eta(\zeta, \xi)|\left(\frac{2-s}{4}\right) A\left(\mathrm{~m}\left|\Psi^{\prime}\left(\frac{\zeta}{\mathrm{m}}\right)\right|,\left|\Psi^{\prime}(\xi)\right|\right) .
\end{aligned}
$$

This completes the proof.

Corollary 5.3. If $\mathrm{m}=\mathrm{s}=1$ in the above Theorem 5.2, then the Theorem 2.1 in [4] is recovered.

Corollary 5.4. Putting $\eta(\zeta, \xi)=\zeta-\xi$ and $m=1$ in the Theorem 5.2, we get inequality (4.1) in [28].

Theorem 5.5. Suppose $\mathcal{J}^{\circ} \subseteq \mathcal{R}$ be an open invex subset with respect to $\eta: \mathcal{J}^{\circ} \times \mathcal{J}^{\circ} \rightarrow \mathcal{R}$ and $\zeta, \xi \in \mathcal{J}^{\circ}$ with $\xi+\eta(\zeta, \xi) \leqslant \xi, q>1, \frac{1}{p}+\frac{1}{q}=1$. Assume that mapping $\Psi: \mathcal{J}^{\circ} \rightarrow \mathcal{R}$ be differentiable such that $\Psi^{\prime} \in$ $\mathrm{L}[\xi+\eta(\zeta, \xi)$, $\xi]$. If $\left|\Psi^{\prime}\right|^{\mathrm{q}}$ is s-type $\mathrm{m}$-preinvex function on $[\xi+\eta(\zeta, \xi)$, $\xi]$ and $\omega \in[0,1], s \in[0,1]$ and $\mathrm{m} \in(0,1]$,

$$
\begin{aligned}
& \left|\frac{\Psi(\xi)+\Psi(\xi+\eta(\zeta, \xi))}{2}-\frac{1}{\eta(\zeta, \xi)} \int_{\xi}^{\xi+\eta(\zeta, \xi)} \Psi(x) d x\right| \\
& \quad \leqslant \frac{|\eta(\zeta, \xi)|}{2}\left(\frac{1}{p+1}\right)^{\frac{1}{p}}\left(\frac{2-s}{2}\right)^{\frac{1}{q}} A^{\frac{1}{q}}\left(m\left|\Psi^{\prime}\left(\frac{\zeta}{m}\right)\right|^{q},\left|\Psi^{\prime}(\xi)\right|^{q}\right),
\end{aligned}
$$

where $A$ is arithmetic mean.

Proof. Assume that for any $\omega \in[0,1]$ and $\zeta, \xi \in \mathcal{J}^{\circ}$, we attain $\xi+\omega \eta(\zeta, \xi) \in \mathcal{J}^{\circ}$. Employing Lemma 5.1, properties of modulus, Hölder's inequality, and definition of $\left|\Psi^{\prime}\right|^{q}$, then we have

$$
\begin{aligned}
& \left|\frac{\Psi(\xi)+\Psi(\xi+\eta(\zeta, \xi))}{2}-\frac{1}{\eta(\zeta, \xi)} \int_{\xi}^{\xi+\eta(\zeta, \xi)} \Psi(x) d x\right| \\
& \quad \leqslant\left|\frac{\eta(\zeta, \xi)}{2} \int_{0}^{1}(1-2 \omega) \Psi^{\prime}(\xi+\omega \eta(\zeta, \xi)) d \omega\right| \\
& \quad \leqslant \frac{|\eta(\zeta, \xi)|}{2}\left(\int_{0}^{1}|1-2 \omega|^{p}\right)^{\frac{1}{p}}\left(\int_{0}^{1}\left|\Psi^{\prime}(\xi+\omega \eta(\zeta, \xi))\right|^{q} d \omega\right)^{\frac{1}{q}} \\
& \quad \leqslant \frac{|\eta(\zeta, \xi)|}{2}\left(\frac{1}{p+1}\right)^{\frac{1}{p}}\left(\left|\Psi^{\prime}(\xi)\right|^{q} \int_{0}^{1}(1-(s \omega)) d \omega+\int_{0}^{1} m\left|\Psi^{\prime}\left(\frac{\zeta}{m}\right)\right|^{q}(1-(s(1-\omega))) d \omega\right)^{\frac{1}{q}} \\
& \quad \leqslant \frac{|\eta(\zeta, \xi)|}{2}\left(\frac{1}{p+1}\right)^{\frac{1}{p}}\left(\frac{2-s}{2}\right)^{\frac{1}{q}} A^{\frac{1}{q}}\left(m\left|\Psi^{\prime}\left(\frac{\zeta}{m}\right)\right|^{q},\left|\Psi^{\prime}(\xi)\right|^{q}\right) .
\end{aligned}
$$

This completes the proof.

Corollary 5.6. If $\mathrm{m}=\mathrm{s}=1$ in Theorem 5.5 , then published Theorem 2.2 in [4] is recovered.

Corollary 5.7. Choosing $\eta(\zeta, \xi)=\zeta-\xi$ and $m=s=1$ in the above Theorem 5.5 , then we get inequality (4.2) in [28]. 
Theorem 5.8. Suppose $\mathcal{J}^{\circ} \subseteq \mathcal{R}$ be an open invex subset with respect to $\eta: \mathcal{J}^{\circ} \times \mathcal{J}^{\circ} \rightarrow \mathcal{R}$ and $\zeta, \xi \in \mathcal{J}^{\circ}$ with $\xi+\eta(\zeta, \xi) \leqslant \xi, q \geqslant 1$. Assume that mapping $\Psi: \mathcal{J}^{\circ} \rightarrow \mathcal{R}$ be differentiable such that $\Psi^{\prime} \in \mathrm{L}[\xi+\eta(\zeta, \xi), \xi]$. If $\left|\Psi^{\prime}\right|^{\mathfrak{q}}$ is s-type $\mathrm{m}$-preinvex function on $[\xi+\eta(\zeta, \xi), \xi]$ and $s \in[0,1], \omega \in[0,1]$ and $\mathrm{m} \in(0,1]$, then

$$
\begin{aligned}
& \left|\frac{\Psi(\xi)+\Psi(\xi+\eta(\zeta, \xi))}{2}-\frac{1}{\eta(\zeta, \xi)} \int_{\xi}^{\xi+\eta(\zeta, \xi)} \Psi(x) d x\right| \\
& \quad \leqslant \frac{|\eta(\zeta, \xi)|}{2}\left(\frac{1}{2}\right)^{1-\frac{2}{q}}\left(\frac{2-s}{4}\right)^{\frac{1}{q}} A^{\frac{1}{q}}\left(m\left|\Psi^{\prime}\left(\frac{\zeta}{m}\right)\right|^{q},\left|\Psi^{\prime}(\xi)\right|^{q}\right),
\end{aligned}
$$

where $A$ is arithmetic mean.

Proof. Assume that for any $\omega \in[0,1]$ and $\zeta, \xi \in \mathcal{J}^{\circ}$, we have $\xi+\omega \eta(\zeta, \xi) \in \mathcal{J}^{\circ}$. Let $q>1$. Applying Lemma 5.1, properties of modulus, power mean inequality, and definition of $\left|\Psi^{\prime}\right|^{q}$, we have

$$
\begin{aligned}
& \left|\frac{\Psi(\xi)+\Psi(\xi+\eta(\zeta, \xi))}{2}-\frac{1}{\eta(\zeta, \xi)} \int_{\xi}^{\xi+\eta(\zeta, \xi)} \Psi(x) d x\right| \\
& \leqslant\left|\frac{\eta(\zeta, \xi)}{2} \int_{0}^{1}(1-2 \omega) \Psi^{\prime}(\xi+\omega \eta(\zeta, \xi)) d \omega\right| \\
& \leqslant \frac{|\eta(\zeta, \xi)|}{2}\left(\int_{0}^{1}|1-2 \omega| \mathrm{d} \omega\right)^{1-\frac{1}{q}}\left(\int_{0}^{1}|1-2 \omega|\left|\Psi^{\prime}(\xi+\omega \eta(\zeta, \xi))\right|^{q} d \omega\right)^{\frac{1}{q}} \\
& \leqslant \frac{|\eta(\zeta, \xi)|}{2}\left(\frac{1}{2}\right)^{1-\frac{1}{q}}\left(\int_{0}^{1}|1-2 \omega|\left[(1-(s \omega))\left|\Psi^{\prime}(\xi)\right|^{q}+(1-(s(1-\omega))) m\left|\Psi^{\prime}\left(\frac{\zeta}{m}\right)\right|^{q}\right] d \omega\right)^{\frac{1}{q}} \\
& \leqslant \frac{|\eta(\zeta, \xi)|}{2}\left(\frac{1}{2}\right)^{1-\frac{1}{q}}\left(\left|\Psi^{\prime}(\xi)\right|^{q} \int_{0}^{1}|1-2 \omega|(1-(s \omega)) d \omega+\int_{0}^{1}|1-2 \omega| m\left|\Psi^{\prime}\left(\frac{\zeta}{m}\right)\right|^{q}(1-(s(1-\omega))) d \omega\right)^{\frac{1}{q}} \\
& =\frac{|\eta(\zeta, \xi)|}{2}\left(\frac{1}{2}\right)^{1-\frac{2}{q}}\left(\frac{2-s}{4}\right)^{\frac{1}{q}} A^{\frac{1}{q}}\left(m\left|\Psi^{\prime}\left(\frac{\zeta}{m}\right)\right|^{q},\left|\Psi^{\prime}(\xi)\right|^{q}\right) \text {. }
\end{aligned}
$$

For the case $\mathrm{q}=1$, we apply the same technique step by step as used in the Theorem 5.2. This completes the proof.

Corollary 5.9. Choosing $\mathrm{m}=\mathrm{s}=1$ in Theorem 5.8 , then

$$
\left|\frac{\Psi(\xi)+\Psi(\xi+\eta(\zeta, \xi))}{2}-\frac{1}{\eta(\zeta, \xi)} \int_{\xi}^{\xi+\eta(\zeta, \xi)} \Psi(x) d x\right| \leqslant \frac{\eta(\zeta, \xi)}{4} A^{\frac{1}{q}}\left[\left|\Psi^{\prime}(\zeta)\right|^{q},\left|\Psi^{\prime}(\xi)\right|^{q}\right],
$$

holds.

Corollary 5.10. Choosing $s=1$ and $\eta(\zeta, \xi)=\zeta-\xi$ in Theorem 5.8, we get inequality (4.3) in [28].

Theorem 5.11. Suppose $\mathcal{J}^{\circ} \subseteq \mathcal{R}$ be an open invex subset with respect to $\eta: \mathcal{J}^{\circ} \times \mathcal{J}^{\circ} \rightarrow \mathcal{R}$ and $\zeta, \xi \in \mathcal{J}^{\circ}$ with $\xi+\eta(\zeta, \xi) \leqslant \xi, q>1, \frac{1}{p}+\frac{1}{q}=1$. Assume that mapping $\Psi: \mathcal{J}^{\circ} \rightarrow \mathcal{R}$ be differentiable such that $\Psi^{\prime} \in$ $\mathrm{L}[\xi+\eta(\zeta, \xi), \xi]$ and $\omega \in[0,1], \mathrm{s} \in[0,1]$ and $\mathrm{m} \in(0,1]$. If $\left|\Psi^{\prime}\right|^{\mathrm{q}}$ be s-type $\mathrm{m}$-preinvex function on $[\xi+\eta(\zeta, \xi), \xi]$, then

$$
\begin{aligned}
& \left|\frac{\Psi(\xi)+\Psi(\xi+\eta(\zeta, \xi))}{2}-\frac{1}{\eta(\zeta, \xi)} \int_{\xi}^{\xi+\eta(\zeta, \xi)} \Psi(x) d x\right| \\
& \leqslant \frac{|\eta(\zeta, \xi)|}{2}\left(\frac{1}{2(p+1)}\right)^{\frac{1}{p}}\left\{\left(m\left|\Psi^{\prime}\left(\frac{\zeta}{m}\right)\right|^{q} \frac{3-2 s}{6}+\left|\Psi^{\prime}(\xi)\right| q^{q} \frac{3-s}{6}\right)^{\frac{1}{q}}+\left(m\left|\Psi^{\prime}\left(\frac{\zeta}{m}\right)\right|^{q} \frac{3-s}{6}+\left|\Psi^{\prime}(\xi)\right|^{q} \frac{3-2 s}{6}\right)^{\frac{1}{q}}\right\} .
\end{aligned}
$$


Proof. Assume that for any $\omega \in[0,1]$ and $\zeta, \xi \in \mathcal{J}^{\circ}$, we have $\xi+\omega \eta(\zeta, \xi) \in \mathcal{J}^{\circ}$. Applying Lemma 5.1, properties of modulus, Hölder-İscan integral inequality, and definition of $\left|\Psi^{\prime}\right|^{q}$, we have

$$
\begin{aligned}
& \left|\frac{\Psi(\xi)+\Psi(\xi+\eta(\zeta, \xi))}{2}-\frac{1}{\eta(\zeta, \xi)} \int_{\xi}^{\xi+\eta(\zeta, \xi)} \Psi(x) d x\right| \\
& \leqslant \frac{|\eta(\zeta, \xi)|}{2} \int_{0}^{1}\left|1-2 \omega \| \Psi^{\prime}(\xi+\omega \eta(\zeta, \xi))\right| d \omega \\
& \leqslant \frac{|\eta(\zeta, \xi)|}{2}\left(\int_{0}^{1}(1-\omega)|1-2 \omega|^{p} d \omega\right)^{\frac{1}{p}}\left(\int_{0}^{1}(1-\omega)\left|\Psi^{\prime}(\xi+\omega \eta(\zeta, \xi))\right|^{q} d \omega\right)^{\frac{1}{q}} \\
& +\frac{|\eta(\zeta, \xi)|}{2}\left(\int_{0}^{1} \omega|1-2 \omega|^{p} \mathrm{~d} \omega\right)^{\frac{1}{p}}\left(\int_{0}^{1} \omega\left|\Psi^{\prime}(\xi+\omega \eta(\zeta, \xi))\right|^{q} \mathrm{~d} \omega\right)^{\frac{1}{q}} \\
& \leqslant \frac{|\eta(\zeta, \xi)|}{2}\left(\frac{1}{2(p+1)}\right)^{\frac{1}{p}}\left(\left|\Psi^{\prime}(\xi)\right|^{q} \int_{0}^{1}(1-\omega)(1-(s \omega)) d \omega+\int_{0}^{1}(1-\omega) m\left|\Psi^{\prime}\left(\frac{\zeta}{m}\right)\right|^{q}(1-(s(1-\omega))) d \omega\right)^{\frac{1}{q}} \\
& +\frac{|\eta(\zeta, \xi)|}{2}\left(\frac{1}{2(p+1)}\right)^{\frac{1}{p}}\left(\left|\Psi^{\prime}(\xi)\right|^{q} \int_{0}^{1} \omega(1-(s \omega)) d \omega+\int_{0}^{1} m\left|\Psi^{\prime}\left(\frac{\zeta}{m}\right)\right|^{q} \omega(1-(s(1-\omega))) d \omega\right)^{\frac{1}{q}} \\
& \leqslant \frac{|\eta(\zeta, \xi)|}{2}\left(\frac{1}{2(p+1)}\right)^{\frac{1}{p}}\left\{\left(m\left|\Psi^{\prime}\left(\frac{\zeta}{m}\right)\right|^{q} \frac{3-2 s}{6}+\left|\Psi^{\prime}(\xi)\right|{ }^{q} \frac{3-s}{6}\right)^{\frac{1}{q}}\right. \\
& \left.+\left(m\left|\Psi^{\prime}\left(\frac{\zeta}{m}\right)\right| q \frac{3-s}{6}+\left.\left|\Psi^{\prime}(\xi)\right|\right|^{q} \frac{3-2 s}{6}\right)^{\frac{1}{q}}\right\} \text {. }
\end{aligned}
$$

This is the required proof.

Corollary 5.12. Choosing $\mathrm{m}=\mathrm{s}=1$ in the above Theorem 5.11 , then

$$
\begin{aligned}
& \left|\frac{\Psi(\xi)+\Psi(\xi+\eta(\zeta, \xi))}{2}-\frac{1}{\eta(\zeta, \xi)} \int_{\xi}^{\xi+\eta(\zeta, \xi)} \Psi(x) d x\right| \\
& \quad \leqslant \frac{|\eta(\zeta, \xi)|}{4}\left(\frac{1}{p+1}\right)^{\frac{1}{p}}\left[\left(\frac{\left|\Psi^{\prime}(\zeta)\right| q}{3}+\frac{2\left|\Psi^{\prime}(\xi)\right| q}{3}\right)^{\frac{1}{q}}+\left(\frac{2\left|\Psi^{\prime}(\zeta)\right|^{\mathfrak{q}}}{3}+\frac{\left|\Psi^{\prime}(\xi)\right|^{\mathfrak{q}}}{3}\right)^{\frac{1}{q}}\right] .
\end{aligned}
$$

Corollary 5.13. Choosing $s=m=1$ and $\eta(\zeta, \xi)=\zeta-\xi$ in Theorem 5.11, we get inequality (4.4) in [28].

Theorem 5.14. Suppose $\mathcal{J}^{\circ} \subseteq \mathcal{R}$ be an open invex subset with respect to $\eta: \mathcal{J}^{\circ} \times \mathcal{J}^{\circ} \rightarrow \mathcal{R}$ and $\zeta, \xi \in \mathcal{J}^{\circ}$ with $\xi+\eta(\zeta, \xi) \leqslant \xi, q \geqslant 1$. Assume that mapping $\Psi: \mathcal{J}^{\circ} \rightarrow \mathcal{R}$ be differentiable such that $\Psi^{\prime} \in \mathrm{L}[\xi+\eta(\zeta, \xi), \xi]$ and $\omega \in[0,1], s \in[0,1]$ and $m \in(0,1]$. If $\left|\Psi^{\prime}\right|^{\mathfrak{a}}$ is s-type $m$-preinvex function on $[\xi+\eta(\zeta, \xi), \xi]$, then

$$
\begin{aligned}
& \left|\frac{\Psi(\xi)+\Psi(\xi+\eta(\zeta, \xi))}{2}-\frac{1}{\eta(\zeta, \xi)} \int_{\xi}^{\xi+\eta(\zeta, \xi)} \Psi(x) d x\right| \\
& \quad \leqslant \frac{|\eta(\zeta, \xi)|}{2}\left(\frac{1}{2}\right)^{2-\frac{2}{q}}\left\{\left(m\left|\Psi^{\prime}\left(\frac{\zeta}{m}\right)\right|{ }^{q} k_{1}(s)+\left|\Psi^{\prime}(\xi)\right|{ }^{q} k_{2}(s)\right)^{\frac{1}{q}}+\left(m\left|\Psi^{\prime}\left(\frac{\zeta}{m}\right)\right|{ }^{q} k_{2}(s)+\left|\Psi^{\prime}(\xi)\right|{ }^{q} k_{1}(s)\right)^{\frac{1}{q}}\right\},
\end{aligned}
$$

where

$$
\begin{aligned}
& \left.k_{1}(s)=\int_{0}^{1}(1-\omega)|1-2 \omega|(1-(s(1-\omega))) d \omega=\int_{0}^{1} \omega|1-2 \omega|(1-(s \omega))\right) d \omega \\
& k_{2}(s)=\int_{0}^{1} \omega|1-2 \omega|(1-(s(1-\omega))) d \omega=\int_{0}^{1}(1-\omega)|1-2 \omega|(1-(s \omega)) d \omega .
\end{aligned}
$$


Proof. Assume that for any $\omega \in[0,1]$ and $\zeta, \xi \in \mathcal{J}^{\circ}$, we have $\xi+\omega \eta(\zeta, \xi) \in \mathcal{J}^{\circ}$. Let $q>1$. Applying Lemma 5.1, properties of modulus, improved power-mean integral inequality, and definition of $\left|\Psi^{\prime}\right|^{q}$, we have

$$
\begin{aligned}
& \left|\frac{\Psi(\xi)+\Psi(\xi+\eta(\zeta, \xi))}{2}-\frac{1}{\eta(\zeta, \xi)} \int_{\xi}^{\xi+\eta(\zeta, \xi)} \Psi(x) d x\right| \\
& \leqslant \frac{|\eta(\zeta, \xi)|}{2} \int_{0}^{1}\left|1-2 \omega \| \Psi^{\prime}(\xi+\omega \eta(\zeta, \xi))\right| \mathrm{d} \omega \\
& \leqslant \frac{|\eta(\zeta, \xi)|}{2}\left(\int_{0}^{1}(1-\omega)|1-2 \omega| \mathrm{d} \omega\right)^{1-\frac{1}{q}}\left(\int_{0}^{1}(1-\omega)|1-2 \omega|\left|\Psi^{\prime}(\xi+\omega \eta(\zeta, \xi))\right|^{q} \mathrm{~d} \omega\right)^{\frac{1}{q}} \\
& +\frac{|\eta(\zeta, \xi)|}{2}\left(\int_{0}^{1}(\omega|1-2 \omega| \mathrm{d} \omega)^{1-\frac{1}{q}}\left(\int_{0}^{1} \omega|1-2 \omega|\left|\Psi^{\prime}(\xi+\omega \eta(\zeta, \xi))\right|^{q} \mathrm{~d} \omega\right)^{\frac{1}{q}}\right. \\
& \leqslant \frac{|\eta(\zeta, \xi)|}{2}\left(\frac{1}{2}\right)^{2-\frac{2}{q}} \\
& \times\left\{\left(\left|\Psi^{\prime}(\xi)\right|^{q} \int_{0}^{1}(1-\omega)|1-2 \omega|(1-(s \omega)) \mathrm{d} \omega+\int_{0}^{1}(1-\omega)|1-2 \omega| \mathrm{m}\left|\Psi^{\prime}\left(\frac{\zeta}{\mathrm{m}}\right)\right|^{\mathbf{q}}(1-(s(1-\omega))) \mathrm{d} \omega\right)^{\frac{1}{q}}\right. \\
& \left.\left.+\left(\left|\Psi^{\prime}(\xi)\right|^{q} \int_{0}^{1} \omega|1-2 \omega|(1-(s \omega))\right) \mathrm{d} \omega+\int_{0}^{1} \omega|1-2 \omega| \mathrm{m}\left|\Psi^{\prime}\left(\frac{\zeta}{\mathrm{m}}\right)\right|^{\mathfrak{q}}(1-(s(1-\omega))) \mathrm{d} \omega\right)^{\frac{1}{q}}\right\} \\
& \leqslant \frac{|\eta(\zeta, \xi)|}{2}\left(\frac{1}{2}\right)^{2-\frac{2}{q}}\left\{\left(m\left|\Psi^{\prime}\left(\frac{\zeta}{m}\right)\right|^{q} k_{1}(s)+\left|\Psi^{\prime}(\xi)\right|{ }^{q} k_{2}(s)\right)^{\frac{1}{q}}+\left(m\left|\Psi^{\prime}\left(\frac{\zeta}{m}\right)\right|{ }^{q} k_{2}(s)+\left|\Psi^{\prime}(\xi)\right|{ }^{q} k_{1}(s)\right)^{\frac{1}{q}}\right\} \text {. }
\end{aligned}
$$

For the case $q=1$, we apply the same technique step by step as used in the Theorem 5.2. This completes the desired proof.

Corollary 5.15. If $\mathrm{m}=\mathrm{s}=1$ in Theorem 5.14, then

$$
\begin{aligned}
& \left|\frac{\Psi(\xi)+\Psi(\xi+\eta(\zeta, \xi))}{2}-\frac{1}{\eta(\zeta, \xi)} \int_{\xi}^{\xi+\eta(\zeta, \xi)} \Psi(x) d x\right| \\
& \quad \leqslant \frac{|\eta(\zeta, \xi)|}{8}\left[\left(\frac{\left|\Psi^{\prime}(\zeta)\right|^{q}}{4}+\frac{3\left|\Psi^{\prime}(\xi)\right|^{q}}{4}\right)^{\frac{1}{q}}+\left(\frac{3\left|\Psi^{\prime}(\zeta)\right|^{q}}{4}+\frac{\left|\Psi^{\prime}(\xi)\right|^{q}}{4}\right)^{\frac{1}{q}}\right] .
\end{aligned}
$$

Corollary 5.16. If we choose $s=m=1$ and $\eta(\zeta, \xi)=\zeta-\xi$ in Theorem 5.14, we get inequality (4.5) in [28].

Note: We pass some comments regarding comparison on the above estimations of the mentioned lemma. Using Lemma 5.1, we established two Theorems 5.5 and 5.11, employing the Hölder and Hölder-İscan inequality. If we compare, Theorem 5.11 gives better result than Theorem 5.5. Similarly, using Lemma 5.1, we estimate two Theorems 5.8 and 5.14, employing power mean inequality and improved power mean inequality. Again, if we compare, Theorem 5.14 gives the better than Theorem 5.8.

Theorem 5.17. Suppose $\mathcal{J}^{\circ} \subseteq \mathcal{R}$ be an open invex subset with respect to $\eta: \mathcal{J}^{\circ} \times \mathcal{J}^{\circ} \rightarrow \mathcal{R}$ and $\zeta, \xi \in \mathcal{J}^{\circ}$ with $\xi+\eta(\zeta, \xi) \leqslant \xi$. Assume that mapping $\Psi: \mathcal{J}^{\circ} \rightarrow \mathcal{R}$ be differentiable such that $\Psi^{\prime} \in \mathrm{L}[\xi+\eta(\zeta, \xi), \xi]$. If $\left|\Psi^{\prime}\right|$ is s-type $(\alpha, m)$-preinvex function on $[\xi+\eta(\zeta, \xi), \xi]$, then

$$
\left|\frac{\Psi(\xi)+\Psi(\xi+\eta(\zeta, \xi))}{2}-\frac{1}{\eta(\zeta, \xi)} \int_{\xi}^{\xi+\eta(\zeta, \xi)} \Psi(x) d x\right| \leqslant \frac{|\eta(\zeta, \xi)|}{2}\left(\Lambda_{1}(s)\left|\Psi^{\prime}(\xi)\right|+m \Lambda_{2}(s)\left|\Psi^{\prime}\left(\frac{\zeta}{m}\right)\right|\right),
$$

holds for $\omega \in[0,1],(\alpha, \mathrm{m}) \in(0,1] \times(0,1]$ and $s \in[0,1]$, where

$$
\begin{aligned}
& \Lambda_{1}(s)=\int_{0}^{1}|1-2 \omega|\left(1-(s \omega)^{\alpha}\right) d \omega=\frac{2 \alpha^{2}+6 \alpha+4-2.2^{-\alpha+1} s^{\alpha}-4 \alpha s^{\alpha}}{4(\alpha+1)(\alpha+2)} \\
& \Lambda_{2}(s)=\int_{0}^{1}|1-2 \omega|\left(1-(s(1-\omega))^{\alpha}\right) d \omega .
\end{aligned}
$$


Proof. Suppose that for any $\omega \in[0,1]$ and $\zeta, \xi \in \mathcal{J}^{\circ}$, we have $\xi+\omega \eta(\zeta, \xi) \in \mathcal{J}^{\circ}$. Applying Lemma 5.1, properties of modulus, and definition of $\left|\Psi^{\prime}\right|$, we have

$$
\begin{aligned}
& \left|\frac{\Psi(\xi)+\Psi(\xi+\eta(\zeta, \xi))}{2}-\frac{1}{\eta(\zeta, \xi)} \int_{\xi}^{\xi+\eta(\zeta, \xi)} \Psi(x) d x\right| \\
& \quad \leqslant\left|\frac{\eta(\zeta, \xi)}{2} \int_{0}^{1}(1-2 \omega) \Psi^{\prime}(\xi+\omega \eta(\zeta, \xi)) d \omega\right| \\
& \left.\quad \leqslant \frac{|\eta(\zeta, \xi)|}{2} \int_{0}^{1}|1-2 \omega|\left(1-(s \omega)^{\alpha}\right)\left|\Psi^{\prime}(\xi)\right|+(1-(s(1-\omega)))^{\alpha} m\left|\Psi^{\prime}\left(\frac{\zeta}{m}\right)\right|\right) d \omega \\
& \quad \leqslant \frac{|\eta(\zeta, \xi)|}{2}\left(\left|\Psi^{\prime}(\xi)\right| \int_{0}^{1}|1-2 \omega|\left(1-(s \omega)^{\alpha}\right) d \omega+m\left|\Psi^{\prime}\left(\frac{\zeta}{m}\right)\right| \int_{0}^{1}|1-2 \omega|(1-(s(1-\omega)))^{\alpha} d \omega\right) \\
& \quad \leqslant \frac{|\eta(\zeta, \xi)|}{2}\left(\Lambda_{1}(s)\left|\Psi^{\prime}(\xi)\right|+m \Lambda_{2}(s)\left|\Psi^{\prime}\left(\frac{\zeta}{m}\right)\right|\right) .
\end{aligned}
$$

This is the required proof.

Corollary 5.18. If we take $\alpha=\mathrm{m}=\mathrm{s}=1$ in the above Theorem 5.17, we attain the published Theorem 2.1 in [4].

Corollary 5.19. Choosing $\eta(\zeta, \xi)=\zeta-\xi$ and $\alpha=m=1$ in the above Theorem 5.17, we get inequality (4.1) in [28].

Theorem 5.20. Suppose $\mathcal{J}^{\circ} \subseteq \mathcal{R}$ be an open invex subset with respect to $\eta: \mathcal{J}^{\circ} \times \mathcal{J}^{\circ} \rightarrow \mathcal{R}$ and $\zeta, \xi \in \mathcal{J}^{\circ}$ with $\xi+\eta(\zeta, \xi) \leqslant \xi, q>1, \frac{1}{p}+\frac{1}{q}=1$. Assume that mapping $\Psi: \mathcal{J}^{\circ} \rightarrow \mathcal{R}$ be differentiable such that $\Psi^{\prime} \in \mathrm{L}[\xi+$ $\eta(\zeta, \xi), \xi]$. If $\left|\Psi^{\prime}\right|^{\mathrm{q}}$ is s-type $(\alpha, \mathrm{m})$-preinvex function on $[\xi+\eta(\zeta, \xi), \xi]$ and $\omega \in[0,1],(\alpha, \mathrm{m}) \in(0,1] \times(0,1]$ and $\mathrm{s} \in[0,1]$, then

$$
\begin{aligned}
& \left|\frac{\Psi(\xi)+\Psi(\xi+\eta(\zeta, \xi))}{2}-\frac{1}{\eta(\zeta, \xi)} \int_{\xi}^{\xi+\eta(\zeta, \xi)} \Psi(x) d x\right| \\
& \quad \leqslant \frac{|\eta(\zeta, \xi)|}{2}\left(\frac{1}{p+1}\right)^{\frac{1}{p}}\left(\Lambda_{3}(s)\left|\Psi^{\prime}(\xi)\right|^{q}+m \Lambda_{4}(s)\left|\Psi^{\prime}\left(\frac{\zeta}{m}\right)\right|^{q}\right),
\end{aligned}
$$

where

$$
\Lambda_{3}(s)=\int_{0}^{1}\left(1-(s \omega)^{\alpha}\right) d \omega=\frac{\alpha+1-s^{\alpha}}{\alpha+1}, \quad \Lambda_{4}(s)=\int_{0}^{1}(1-(s(1-\omega)))^{\alpha} \mathrm{d} \omega=\frac{-(1-s)^{\alpha+1}-1}{s(\alpha+1)} .
$$

Proof. Suppose that for any $\omega \in[0,1]$ and $\zeta, \xi \in \mathcal{J}^{\circ}$, we have $\xi+\omega \eta(\zeta, \xi) \in \mathcal{J}^{\circ}$. Applying Lemma 5.1, properties of modulus, Hölder's integral inequality, and definition of $\left|\Psi^{\prime}\right|^{q}$, we have

$$
\begin{aligned}
& \left|\frac{\Psi(\xi)+\Psi(\xi+\eta(\zeta, \xi))}{2}-\frac{1}{\eta(\zeta, \xi)} \int_{\xi}^{\xi+\eta(\zeta, \xi)} \Psi(x) d x\right| \\
& \leqslant\left|\frac{\eta(\zeta, \xi)}{2} \int_{0}^{1}(1-2 \omega) \Psi^{\prime}(\xi+\omega \eta(\zeta, \xi)) d \omega\right| \\
& \quad \leqslant \frac{|\eta(\zeta, \xi)|}{2}\left(\int_{0}^{1}|1-2 \omega|^{p}\right)^{\frac{1}{p}}\left(\int_{0}^{1}\left|\Psi^{\prime}(\xi+\omega \eta(\zeta, \xi))\right|^{q} d \omega\right)^{\frac{1}{q}} \\
& \quad \leqslant \frac{|\eta(\zeta, \xi)|}{2}\left(\frac{1}{p+1}\right)^{\frac{1}{p}}\left(\left|\Psi^{\prime}(\xi)\right|^{q} \int_{0}^{1}\left(1-(s \omega)^{\alpha}\right) d \omega+\int_{0}^{1} m\left|\Psi^{\prime}\left(\frac{\zeta}{m}\right)\right|^{q}(1-(s(1-\omega)))^{\alpha} d \omega\right)^{\frac{1}{q}} \\
& \quad \leqslant \frac{|\eta(\zeta, \xi)|}{2}\left(\frac{1}{p+1}\right)^{\frac{1}{p}}\left(\Lambda_{3}(s)\left|\Psi^{\prime}(\xi)\right|^{q}+m \Lambda_{4}(s)\left|\Psi^{\prime}\left(\frac{\zeta}{m}\right)\right|^{q}\right) .
\end{aligned}
$$

This is the required proof. 
Corollary 5.21. Choosing $\alpha=m=s=1$ in the above Theorem 5.20, we attain the published Theorem 2.2 in [4].

Corollary 5.22. Choosing $\eta(\zeta, \xi)=\zeta-\xi$ and $\alpha=m=s=1$ in the above Theorem 5.20, then we get inequality (4.2) in [28].

Theorem 5.23. Suppose $\mathcal{J}^{\circ} \subseteq \mathcal{R}$ be an open invex subset with respect to $\eta: \mathcal{J}^{\circ} \times \mathcal{J}^{\circ} \rightarrow \mathcal{R}$ and $\zeta, \xi \in \mathcal{J}^{\circ}$ with $\xi+\eta(\zeta, \xi) \leqslant \xi, q \geqslant 1$. Assume that mapping $\Psi: \mathcal{J}^{\circ} \rightarrow \mathcal{R}$ be differentiable such that $\Psi^{\prime} \in \mathrm{L}[\xi+\eta(\zeta, \xi), \xi]$. If $\left|\Psi^{\prime}\right|^{\mathfrak{q}}$ is s-type $(\alpha, \mathrm{m})$-preinvex function on $[\xi+\eta(\zeta, \xi), \xi]$, then

$$
\begin{aligned}
& \left|\frac{\Psi(\xi)+\Psi(\xi+\eta(\zeta, \xi))}{2}-\frac{1}{\eta(\zeta, \xi)} \int_{\xi}^{\xi+\eta(\zeta, \xi)} \Psi(x) d x\right| \\
& \leqslant \frac{|\eta(\zeta, \xi)|}{2}\left(\frac{1}{2}\right)^{1-\frac{2}{q}}\left(\Lambda_{1}(s)\left|\Psi^{\prime}(\xi)\right|^{q}+m \Lambda_{2}(s)\left|\Psi^{\prime}\left(\frac{\zeta}{m}\right)\right|^{q}\right),
\end{aligned}
$$

holds for $\omega \in[0,1],(\alpha, m) \in(0,1] \times(0,1]$ and $s \in[0,1]$, where $\Lambda_{1}(s)$ and $\Lambda_{2}(s)$ are defined in Theorem 5.17 .

Proof. Assume that for any $\omega \in[0,1]$ and $\zeta, \xi \in \mathcal{J}^{\circ}$, we have $\xi+\omega \eta(\zeta, \xi) \in \mathcal{J}^{\circ}$. Let $q>1$. Applying Lemma 5.1, properties of modulus, power mean inequality, and definition of $\left|\Psi^{\prime}\right|^{q}$, we have

$$
\begin{aligned}
& \left|\frac{\Psi(\xi)+\Psi(\xi+\eta(\zeta, \xi))}{2}-\frac{1}{\eta(\zeta, \xi)} \int_{\xi}^{\xi+\eta(\zeta, \xi)} \Psi(x) d x\right| \\
& \leqslant\left|\frac{\eta(\zeta, \xi)}{2} \int_{0}^{1}(1-2 \omega) \Psi^{\prime}(\xi+\omega \eta(\zeta, \xi)) d \omega\right| \\
& \leqslant \frac{|\eta(\zeta, \xi)|}{2}\left(\int_{0}^{1}|1-2 \omega| d \omega\right)^{1-\frac{1}{q}}\left(\int_{0}^{1}|1-2 \omega|\left|\Psi^{\prime}(\xi+\omega \eta(\zeta, \xi))\right|^{q} d \omega\right)^{\frac{1}{q}} \\
& \left.\leqslant \frac{|\eta(\zeta, \xi)|}{2}\left(\frac{1}{2}\right)^{1-\frac{1}{q}}\left(\int_{0}^{1}|1-2 \omega|\left(1-(s \omega)^{\alpha}\right)\left|\Psi^{\prime}(\xi)\right|^{q}+(1-(s(1-\omega)))^{\alpha} m\left|\Psi^{\prime}\left(\frac{\zeta}{m}\right)\right|^{q}\right] d \omega\right)^{\frac{1}{q}} \\
& \leqslant \frac{|\eta(\zeta, \xi)|}{2}\left(\frac{1}{2}\right)^{1-\frac{1}{q}}\left(\left|\Psi^{\prime}(\xi)\right|^{q} \int_{0}^{1}|1-2 \omega|\left(1-(s \omega)^{\alpha}\right) d \omega+\int_{0}^{1}|1-2 \omega| m^{\prime}\left|\Psi^{\prime}\left(\frac{\zeta}{m}\right)\right|^{q}\left(1-(s(1-\omega))^{\alpha}\right) d \omega\right)^{\frac{1}{q}} \\
& =\frac{|\eta(\zeta, \xi)|}{2}\left(\frac{1}{2}\right)^{1-\frac{2}{q}}\left(\Lambda_{1}(s)\left|\Psi^{\prime}(\xi)\right|^{q}+m \Lambda_{2}(s)\left|\Psi^{\prime}\left(\frac{\zeta}{m}\right)\right|^{q}\right) .
\end{aligned}
$$

For the case $q=1$, we apply the same technique step by step as used in the Theorem 5.17. This completes the proof.

Corollary 5.24. Choosing $\alpha=m=s=1$ in the above Theorem 5.23, then

$$
\left|\frac{\Psi(\xi)+\Psi(\xi+\eta(\zeta, \xi))}{2}-\frac{1}{\eta(\zeta, \xi)} \int_{\xi}^{\xi+\eta(\zeta, \xi)} \Psi(x) d x\right| \leqslant \frac{\eta(\zeta, \xi)}{4} A^{\frac{1}{q}}\left[\left|\Psi^{\prime}(\zeta)\right|^{q},\left|\Psi^{\prime}(\xi)\right|^{q}\right] .
$$

Corollary 5.25. If $\alpha=m=s=1$ and $\eta(\zeta, \xi)=\zeta-\xi$ in Theorem 5.23, we get inequality (4.3) in [28].

Theorem 5.26. Suppose $\mathcal{J}^{\circ} \subseteq \mathcal{R}$ be an open invex subset with respect to $\eta: \mathcal{J}^{\circ} \times \mathcal{J}^{\circ} \rightarrow \mathcal{R}$ and $\zeta, \xi \in \mathcal{J}^{\circ}$ with $\xi+\eta(\zeta, \xi) \leqslant \xi, q>1, \frac{1}{\mathrm{p}}+\frac{1}{\mathrm{q}}=1$. Assume that mapping $\Psi: \mathcal{J}^{\circ} \rightarrow \mathcal{R}$ be differentiable such that $\Psi^{\prime} \in$ $\mathrm{L}[\xi+\eta(\zeta, \xi), \xi]$. If $\left|\Psi^{\prime}\right|^{\mathrm{a}}$ be s-type $(\alpha, \mathrm{m})$-preinvex function on $[\xi+\eta(\zeta, \xi), \xi]$, then

$$
\begin{aligned}
& \left|\frac{\Psi(\xi)+\Psi(\xi+\eta(\zeta, \xi))}{2}-\frac{1}{\eta(\zeta, \xi)} \int_{\xi}^{\xi+\eta(\zeta, \xi)} \Psi(x) d x\right| \\
& \leqslant \frac{|\eta(\zeta, \xi)|}{2}\left(\frac{1}{2(p+1)}\right)^{\frac{1}{p}}\left\{\left(\Lambda_{5}(s)\left|\Psi^{\prime}(\xi)\right|^{q}+m \Lambda_{6}(s)\left|\Psi^{\prime}\left(\frac{\zeta}{m}\right)\right|^{q}\right)^{\frac{1}{q}}+\left(\Lambda_{7}(s)\left|\Psi^{\prime}(\xi)\right|^{q}+m \Lambda_{8}(s)\left|\Psi^{\prime}\left(\frac{\zeta}{m}\right)\right|^{q}\right)^{\frac{1}{q}}\right\},
\end{aligned}
$$


holds for $\omega \in[0,1],(\alpha, \mathrm{m}) \in(0,1] \times(0,1]$ and $s \in[0,1]$, where

$$
\begin{aligned}
& \Lambda_{5}(s)=\int_{0}^{1}(1-\omega)\left(1-(s \omega)^{\alpha}\right) d \omega=\frac{1}{2}-\frac{s^{\alpha}}{(\alpha+1)(\alpha+2)} \\
& \Lambda_{6}(s)=\int_{0}^{1}(1-\omega)(1-(s(1-\omega)))^{\alpha} d \omega=\frac{(1-s)^{\alpha+2}-1-\left((1-s)^{\alpha+1}-(\alpha+1)\right) \frac{\alpha+2}{\alpha+1}}{s^{2}(\alpha+2)} \\
& \Lambda_{7}(s)=\int_{0}^{1} \omega\left(1-(s \omega)^{\alpha}\right) d \omega=\frac{\alpha+2-2 s^{\alpha}}{2(\alpha+2)}, \\
& \Lambda_{6}(s)=\int_{0}^{1} \omega(1-(s(1-\omega)))^{\alpha} \mathrm{d} \omega=-\frac{(1-s)^{\alpha+2}-1-(1-s)\left[1-s^{\alpha+1}-(\alpha+1)\right] \frac{\alpha+2}{\alpha+1}}{s^{2}(\alpha+2)} .
\end{aligned}
$$

Proof. Assume that for any $\omega \in[0,1]$ and $\zeta, \xi \in \mathcal{J}^{\circ}$, we have $\xi+\omega \eta(\zeta, \xi) \in \mathcal{J}^{\circ}$. Applying Lemma 5.1, properties of modulus, Hölder-İscan integral inequality, and definition of $\left|\Psi^{\prime}\right| \mathrm{q}$, we have

$$
\begin{aligned}
& \left|\frac{\Psi(\xi)+\Psi(\xi+\eta(\zeta, \xi))}{2}-\frac{1}{\eta(\zeta, \xi)} \int_{\xi}^{\xi+\eta(\zeta, \xi)} \Psi(x) d x\right| \\
& \leqslant \frac{|\eta(\zeta, \xi)|}{2} \int_{0}^{1}|1-2 \omega|\left|\Psi^{\prime}(\xi+\omega \eta(\zeta, \xi))\right| \mathrm{d} \omega \\
& \leqslant \frac{|\eta(\zeta, \xi)|}{2}\left(\int_{0}^{1}(1-\omega)|1-2 \omega|^{p} \mathrm{~d} \omega\right)^{\frac{1}{p}}\left(\int_{0}^{1}(1-\omega)\left|\Psi^{\prime}(\xi+\omega \eta(\zeta, \xi))\right|^{q} d \omega\right)^{\frac{1}{q}} \\
& +\frac{|\eta(\zeta, \xi)|}{2}\left(\int_{0}^{1} \omega|1-2 \omega|^{p} \mathrm{~d} \omega\right)^{\frac{1}{p}}\left(\int_{0}^{1} \omega\left|\Psi^{\prime}(\xi+\omega \eta(\zeta, \xi))\right|^{q} d \omega\right)^{\frac{1}{q}} \\
& \leqslant \frac{|\eta(\zeta, \xi)|}{2}\left(\frac{1}{2(p+1)}\right)^{\frac{1}{p}}\left(\left|\Psi^{\prime}(\xi)\right|^{q} \int_{0}^{1}(1-\omega)\left(1-(s \omega)^{\alpha}\right) d \omega+\int_{0}^{1}(1-\omega) m\left|\Psi^{\prime}\left(\frac{\zeta}{m}\right)\right|^{q}(1-(s(1-\omega)))^{\alpha} d \omega\right)^{\frac{1}{q}} \\
& +\frac{|\eta(\zeta, \xi)|}{2}\left(\frac{1}{2(p+1)}\right)^{\frac{1}{p}}\left(\left|\Psi^{\prime}(\xi)\right|^{q} \int_{0}^{1} \omega\left(1-(s \omega)^{\alpha}\right) d \omega+\int_{0}^{1} m\left|\Psi^{\prime}\left(\frac{\zeta}{m}\right)\right|^{q} \omega(1-(s(1-\omega)))^{\alpha} d \omega\right)^{\frac{1}{q}} \\
& \leqslant \frac{|\eta(\zeta, \xi)|}{2}\left(\frac{1}{2(p+1)}\right)^{\frac{1}{p}}\left\{\left(\Lambda_{5}(s)\left|\Psi^{\prime}(\xi)\right|^{q}+m \Lambda_{6}(s)\left|\Psi^{\prime}\left(\frac{\zeta}{m}\right)\right|^{q}\right)^{\frac{1}{q}}+\left(\Lambda_{7}(s)\left|\Psi^{\prime}(\xi)\right|^{q}+m \Lambda_{8}(s)\left|\Psi^{\prime}\left(\frac{\zeta}{m}\right)\right|^{q}\right)^{\frac{1}{q}}\right\} .
\end{aligned}
$$

This completes the desired proof.

Corollary 5.27. If we take $s=\alpha=m=1$ and $\eta(\zeta, \xi)=\zeta-\xi$ in Theorem 5.26, we get inequality (4.4) in [28].

Theorem 5.28. Suppose $\mathcal{J}^{\circ} \subseteq \mathcal{R}$ be an open invex subset with respect to $\eta: \mathcal{J}^{\circ} \times \mathcal{J}^{\circ} \rightarrow \mathcal{R}$ and $\zeta, \xi \in \mathcal{J}^{\circ}$ with $\xi+\eta(\zeta, \xi) \leqslant \xi, q \geqslant 1$. Assume that mapping $\Psi: \mathcal{J}^{\circ} \rightarrow \mathcal{R}$ be differentiable such that $\Psi^{\prime} \in \mathrm{L}[\xi+\eta(\zeta, \xi), \xi]$. If $\left|\Psi^{\prime}\right|^{q}$ is s-type $(\alpha, m)$-preinvex function on $[\xi+\eta(\zeta, \xi), \xi]$, then

$$
\begin{aligned}
& \left|\frac{\Psi(\xi)+\Psi(\xi+\eta(\zeta, \xi))}{2}-\frac{1}{\eta(\zeta, \xi)} \int_{\xi}^{\xi+\eta(\zeta, \xi)} \Psi(x) d x\right| \\
& \quad \leqslant \frac{|\eta(\zeta, \xi)|}{2}\left(\frac{1}{2}\right)^{2-\frac{2}{q}}\left\{\left(\Lambda_{9}(s)\left|\Psi^{\prime}(\xi)\right|^{q}+m \Lambda_{10}(s)\left|\Psi^{\prime}\left(\frac{\zeta}{m}\right)\right|^{q}\right)^{\frac{1}{q}}+\left(\Lambda_{11}(s)\left|\Psi^{\prime}(\xi)\right|^{q}+m \Lambda_{12}(s)\left|\Psi^{\prime}\left(\frac{\zeta}{m}\right)\right|^{q}\right)^{\frac{1}{q}}\right\},
\end{aligned}
$$

holds for $\omega \in[0,1],(\alpha, \mathrm{m}) \in(0,1] \times(0,1]$ and $\mathrm{s} \in[0,1]$, where

$$
\begin{array}{ll}
\Lambda_{9}(s)=\int_{0}^{1} \omega|1-2 \omega|\left(1-(s \omega)^{\alpha}\right) d \omega, & \Lambda_{10}(s)=\int_{0}^{1}(1-\omega)|1-2 \omega|(1-(s(1-\omega)))^{\alpha} \mathrm{d} \omega, \\
\Lambda_{11}(s)=\int_{0}^{1} \omega|1-2 \omega|\left(1-(s \omega)^{\alpha}\right) d \omega, & \Lambda_{12}(s)=\int_{0}^{1} \omega|1-2 \omega|(1-(s(1-\omega)))^{\alpha} \mathrm{d} \omega .
\end{array}
$$


Proof. Assume that for any $\omega \in[0,1]$ and $\zeta, \xi \in \mathcal{J}^{\circ}$, we have $\xi+\omega \eta(\zeta, \xi) \in \mathcal{J}^{\circ}$. Let $q>1$. From lemma 5.1, properties of modulus, improved power-mean integral inequality, and definition of $\left|\Psi^{\prime}\right|^{q}$, we have

$$
\begin{aligned}
& \left|\frac{\Psi(\xi)+\Psi(\xi+\eta(\zeta, \xi))}{2}-\frac{1}{\eta(\zeta, \xi)} \int_{\xi}^{\xi+\eta(\zeta, \xi)} \Psi(x) \mathrm{d} x\right| \\
& \leqslant \frac{|\eta(\zeta, \xi)|}{2} \int_{0}^{1}\left|1-2 \omega \| \Psi^{\prime}(\xi+\omega \eta(\zeta, \xi))\right| \mathrm{d} \omega \\
& \leqslant \frac{|\eta(\zeta, \xi)|}{2}\left(\int_{0}^{1}(1-\omega)|1-2 \omega| \mathrm{d} \omega\right)^{1-\frac{1}{q}}\left(\int_{0}^{1}(1-\omega)\left|1-2 \omega \| \Psi^{\prime}(\xi+\omega \eta(\zeta, \xi))\right|^{\mathrm{q}} \mathrm{d} \omega\right)^{\frac{1}{q}} \\
& +\frac{|\eta(\zeta, \xi)|}{2}\left(\int_{0}^{1}(\omega|1-2 \omega| \mathrm{d} \omega)^{1-\frac{1}{q}}\left(\int_{0}^{1} \omega|1-2 \omega|\left|\Psi^{\prime}(\xi+\omega \eta(\zeta, \xi))\right|^{q} \mathrm{~d} \omega\right)^{\frac{1}{q}}\right. \\
& \leqslant \frac{|\eta(\zeta, \xi)|}{2}\left(\frac{1}{2}\right)^{2-\frac{2}{9}} \\
& \times\left\{\left(\left|\Psi^{\prime}(\xi)\right|^{q} \int_{0}^{1}(1-\omega)|1-2 \omega|\left(1-(s \omega)^{\alpha}\right) \mathrm{d} \omega+\int_{0}^{1}(1-\omega)|1-2 \omega| \mathrm{m}\left|\Psi^{\prime}\left(\frac{\zeta}{\mathrm{m}}\right)\right|^{\mathrm{q}}(1-(s(1-\omega)))^{\alpha} \mathrm{d} \omega\right)^{\frac{1}{q}}\right. \\
& +\left(\left|\Psi^{\prime}(\xi)\right|^{\mid} \int_{0}^{1} \omega|1-2 \omega|\left(1-(s \omega)^{\alpha}\right) \mathrm{d} \omega+\int_{0}^{1} \omega|1-2 \omega| \mathrm{m}\left|\Psi^{\prime}\left(\frac{\zeta}{\mathrm{m}}\right)\right|^{\mathfrak{q}}\left(1-(s(1-\omega))^{\alpha} \mathrm{d} \omega\right)^{\frac{1}{q}}\right\} \\
& \leqslant \frac{|\eta(\zeta, \xi)|}{2}\left(\frac{1}{2}\right)^{2-\frac{2}{q}}\left\{\left(\Lambda_{9}(s)\left|\Psi^{\prime}(\xi)\right|^{q}+m \Lambda_{10}(s)\left|\Psi^{\prime}\left(\frac{\zeta}{m}\right)\right|^{q}\right)^{\frac{1}{q}}+\left(\Lambda_{11}(s)\left|\Psi^{\prime}(\xi)\right|^{q}+m \Lambda_{12}(s)\left|\Psi^{\prime}\left(\frac{\zeta}{m}\right)\right|^{q}\right)^{\frac{1}{q}}\right\} .
\end{aligned}
$$

For the case $\mathrm{q}=1$, we apply the same technique step by step as used in the Theorem 5.17. This completes the proof.

Corollary 5.29. If we take $\alpha=\mathrm{m}=1$ and $\mathrm{s}=1$ in Theorem 5.28 , then

$$
\left|\frac{\Psi(\xi)+\Psi(\xi+\eta(\zeta, \xi))}{2}-\frac{1}{\eta(\zeta, \xi)} \int_{\xi}^{\xi+\eta(\zeta, \xi)} \Psi(x) d x\right| \leqslant \frac{|\eta(\zeta, \xi)|}{8}\left[\left(\frac{\left|\Psi^{\prime}(\zeta)\right|^{q}}{4}+\frac{3\left|\Psi^{\prime}(\xi)\right|^{q}}{4}\right)^{\frac{1}{q}}+\left(\frac{3\left|\Psi^{\prime}(\zeta)\right|^{q}}{4}+\frac{\left|\Psi^{\prime}(\xi)\right|^{q}}{4}\right)^{\frac{1}{q}}\right] .
$$

Corollary 5.30. If we take $\mathrm{s}=\alpha=\mathrm{m}=1$ and $\eta(\zeta, \xi)=\zeta-\xi$ in Theorem 5.28, we get inequality (4.5) in [28].

Note: We pass some comments regarding comparison on the above estimations. Using Lemma 5.1, we established Theorems 5.20 and 5.26, with the help of Hölder and Hölder-İscan inequality. If we compare, then Theorem 5.26 gives the better result than Theorem 5.20. Similarly, Using Lemma 5.1, we presented Theorems 5.23 and 5.28, with the help of power mean inequality and improved power mean inequality. If we compare, then Theorem 5.28 gives the better result than Theorem 5.23.

\section{Conclusion}

In our article, we have defined two new preinvex functions namely s-type m-preinvex and s-type $(\alpha, m)$-preinvexity and also discussed some related algebraic properties. Next, employing these new notions of preinvexity, we presented some novel variant of $\mathrm{H}-\mathrm{H}$ type inequality and some of its refinements. To support their summed up behaviour, we show the association between our results and the generally established ones in the literature. In future, researchers can extend this concept in variant ways like time scale calculus, Raina convexity, polynomial Raina convexity, quantum calculus, fractional calculus, etc. We believe the procedure of this manuscript will motivate mathematicians to investigate a more amazing sequel in this direction.

\section{Acknowledgment}

The authors are grateful to the referees for numerous comments that improved the quality of the paper. 


\section{References}

[1] H. Ahmad, M. Tariq, S. K. Sahoo, J. Baili, C. Cesarano, New estimations of Hermite-Hadamard type integral inequalities for special functions, Fractal Fract., 5 (2021), 26 pages. 1

[2] T. Antczak, Mean value in invexity analysis, Nonlinear Anal., 60 (2005), 1473-1484. 2

[3] M. U. Awan, S. Talib, M. A. Noor, Y.-M. Chu, K. I. Noor, Some trapezium-like inequalities involving functions having strongly n-polynomial preinvexity property of higher order, J. Funct. Spaces, 2020 (2020), 9 pages. 2

[4] G. Barani, Ghazanfari, S. S. Dragomir, Hermite-Hadamard inequality for functions whose derivatives absolute values are preinvex, J. Inequal. Appl., 2012 (2012), 9 pages. 2, 5.1, 5.3, 5.6, 5.18, 5.21

[5] S. I. Butt, A. Kashuri, M. Tariq, J. Nasir, A. Aslam, W. Geo, Hermite-Hadamard-type inequalities via n-polynomial exponential-type convexity and their applications, Adv. Difference Equ., 2020 (2020), 25 pages. 1

[6] S. I. Butt, A. Kashuri, M. Tariq, J. Nasir, A. Aslam, W. Geo, n-polynomial exponential-type p-convex function with some related inequalities and their application, Heliyon, 6 (2020), 9 pages.

[7] S. I. Butt, M. Nadeem, S. Qaisar, A. O. Akdemir, T. Abdeljawad, Hermite-Jensen-Mercer type inequalities for conformable integrals and related results, Adv. Difference Equ., 2020 (2020), 24 pages.

[8] S. I. Butt, M. Tariq, A. Aslam, H. Ahmad, T. A. Nofel, Hermite-Hadamard type inequalities via generalized harmonic exponential convexity and applications, J. Funct. Spaces, 2021 (2021), 12 pages. 1

[9] Y. P. Deng, H. Kalsoom, S. H. Wu, Some new Quantum Hermite-Hadamard-type estimates within a class of generalized $(\mathrm{s}, \mathrm{m})$-preinvex functions, Symmetry, 11 (2019), 15 pages. 1

[10] T.-S. Du, J.-G. Liao, Y.-J. Li, Properties and integral inequalities of Hadamard-Simpson type for the generalized (s, $\mathrm{m})-$ preinvex functions, J. Nonlinear Sci. Appl., 9 (2016), 3112-3126. 2

[11] J. Hadamard, Étude sur les propriétés des fonctions entiéres et en particulier d'une fonction consid'erée par Riemann, J. Math. Pures Appl., 58 (1893), 171-215. 4.2

[12] M. A. Khan, Y. M. Chu, T. U. Khan, J. Khan, Some new inequalities of Hermite-Hadamard type for s-convex functions with applications, Open Math., 15 (2017), 1414-1430. 1

[13] S. Kumari, M. Kumari, R. Chugh, Generation of new fractals via SP orbit with s-convexity, Int. J. Eng. Technol., 9 (2017), 2491-2504. 1

[14] Y. C. Kwun, A. A. Shahid, W. Nazeer, M. Abbas, S. M. Kang, Fractal generation via CR iteration scheme with sconvexity, IEEE Access, 7 (2019), 69986-69997. 1

[15] M. A. Latif, M. Shoaib, Hermite-Hadamard type integral inequalities for differentiable m-preinvex and $(\alpha, \mathrm{m})$-preinvex functions, J. Egyptian Math. Soc., 23 (2015), 236-241. 1, 2

[16] S. Mititelu, Invex sets, Stud. Cerc. Mat., 46 (1994), 529-532. 2, 2

[17] S. R. Mohan, S. K. Neogy, On invex sets and preinvex functions, J. Math. Anal. Appl., 189 (1995), 901-908. 2

[18] C. P. Niculescu, L.-E. Persson, Convex functions and their applications, Springer-Verlag, New York, (2006). 1, iii

[19] M. A. Noor, On Hadamard integral inequalities invoving two log-preinvex functions, J. Inequal. Pure. Appl. Math., 8 (2007), 14 pages. 2

[20] M. A. Noor, Hadamard integral inequalities for product of two preinvex function, Nonlinear Anal. Forum, 14 (2009), 167-173. 2

[21] M. A. Noor, K. I. Noor, M. U. Awan, J. Y. Li, On Hermite-Hadamard inequalities for h-preinvex functions, Filomat, 28 (2014), 1463-1474. 2

[22] S. Özcan, İ. İşcan, Some new Hermite-Hadamard type integral inequalities for the s-convex functions and theirs applications, J. Inequal. Appl., 201 (2019), 1-14. 1

[23] S. Rashid, İ. İşcan, D. Baleanu, Y.-M. Chu, Generation of new fractional inequalities via n-polynomials s-type convexity with applications, Adv. Difference Equ., 2020 (2020), 20 pages. 1, 2, ii

[24] S. K. Sahoo, H. Ahmad, M. Tariq, B. Kodamasingh, H. Aydi, M. De la Sen, Hermite-Hadamard type inequalities involving $k$-fractional operator for $(\overline{\mathrm{h}}, \mathrm{m})$-convex Functions, Symmetry, 13 (2021), 16 pages. 1

[25] S. K. Sahoo, M. Tariq, H. Ahmad, J. Nasir, H. Aydi, A. Mukheimer, New Ostrowski-type fractional integral inequalities via generalized exponential-type convex functions and applications, Symmetry, 13 (2021), 18 pages. 1

[26] H. J. Skala, On the characterization of certain similarly ordered super-additive functionals, Proc. Amer. Math. Soc., 126 (1998), 1349-1353. 2

[27] G. H. Toader, Some generalizations of the convexity, Proc. Colloq. Approx. Optim (Cluj-Napoca, Romania), 1985 (1985), 329-338. 1

[28] T. Toplu, M. Kadakal, Í. Íşcan, On n-polynomial convexity and some related inequalities, AIMS Math., 5 (2020), 13041318. 5.4, 5.7, 5.10, 5.13, 5.16, 5.19, 5.22, 5.25, 5.27, 5.30

[29] T. Weir, B. Mond, Preinvex functions in multiple objective optimization, J. Math. Anal. Appl., 136 (1988), 29-38. 2, i

[30] B.-Y. Xi, F. Qi, Some integral inequalities of Hermite-Hadamard type for convex functions with applications to means, J. Funct. Spaces Appl., 2012 (2012), 14 pages. 1 